\title{
Differences in the Sub-seasonal Predictability of Extreme Stratospheric Events
}

\author{
Rachel W.-Y. Wu ${ }^{1}$, Zheng $\mathrm{Wu}^{1}$, and Daniela I.V. Domeisen ${ }^{1,2}$ \\ ${ }^{1}$ ETH Zurich, Zurich, Switzerland \\ ${ }^{2}$ University of Lausanne, Lausanne, Switzerland
}

Correspondence: Rachel Wai-Ying Wu (rachel.wu@env.ethz.ch)

\begin{abstract}
Extreme stratospheric events such as sudden stratospheric warming and strong vortex events associated with an anomalously weak or strong polar vortex can have downward impacts on surface weather that can last for several weeks to months. Hence, successful predictions of these stratospheric events would be beneficial for extended range weather prediction. However, the predictability limit of extreme stratospheric events is most often limited to around 2 weeks or less. The predictability also strongly differs between events, and between event types. The reasons for the observed differences in the predictability, however, are not resolved. To better understand the predictability differences between events, we expand the definitions of extreme stratospheric events to wind deceleration and acceleration events, and conduct a systematic comparison of predictability between event types in the European Centre for Medium-Range Weather Forecasts (ECMWF) prediction system for the sub-seasonal predictions. We find that wind deceleration and acceleration events follow the same predictability behaviour, that is, events of stronger magnitude are less predictable in a close to linear relationship, to the same extent for both types of events. There are however deviations from this linear behaviour for very extreme events. The difficulties of the prediction system in predicting extremely strong anomalies can be traced to a poor predictability of extreme wave activity pulses in the lower stratosphere, which impacts the prediction of deceleration events, and interestingly, also acceleration events. Improvements in the understanding of the wave amplification that is associated with extremely strong wave activity pulses and accurately representing these processes in the model is expected to enhance the predictability of stratospheric extreme events and, by extension, their impacts on surface weather and climate.
\end{abstract}

\section{Introduction}

The stratospheric polar vortex (SPV) is a band of strong westerly winds over the polar region at the height of around 20-50km during winter. These circumpolar winds result from a strong temperature gradient in the stratosphere between the polar and subtropical regions during winter due to reduced solar heating over the polar regions. As westerly flow in the stratosphere favours upward wave propagation (Charney and Drazin, 1961), planetary-scale waves formed at the troposphere can propagate 
https://doi.org/10.5194/wcd-2021-84

Preprint. Discussion started: 11 January 2022

(c) Author(s) 2022. CC BY 4.0 License.

(c) (i)

Weather and

Climate Dynamics

Discussions

upwards into the stratosphere (e.g. Polvani and Waugh, 2004; Sjoberg and Birner, 2012). Depending on the wave activity and the state of the vortex, the SPV can undergo periods of weakening or strengthening, thus largely varying in strength during the wintertime.

The weakening and strengthening of the SPV can be understood in the framework of wave-mean flow interaction (Matsuno, 1970; Holton and Mass, 1976). Before vortex weakening events, anomalously strong wave activity is observed in the lower stratosphere (Polvani and Waugh, 2004; Hinssen and Ambaum, 2010). The waves can precondition the vortex via wave breaking (Limpasuvan et al., 2004; Albers and Birner, 2014), shaping the vortex structure to be more favourable for upward wave propagation. A preconditioned vortex is associated with a region of large and positive refractive index (Matsuno, 1970; Simpson et al., 2009; Karoly and Hoskins, 1982). As the refractive index for stationary planetary waves is proportional to the meridional potential vorticity (PV) gradient, the meridional PV gradient can be used as a proxy for waveguidability (Albers and Birner, 2014; Jucker and Reichler, 2018). On the contrary, when wave activity is weak and the SPV is relatively undisturbed, radiative cooling takes place due to the strong latitudinal temperature gradient induced by the lack of insolation during polar night around the pole. The vortex thus strengthens on radiative timescales (Limpasuvan et al., 2005; Hitchcock and Shepherd, 2013). Holton and Mass (1976) demonstrated using a simple mechanistic model that when the wave forcing is below a critical level, the vortex accelerates and approaches a state close to radiative equilibrium.

There exist various definitions to characterise the weak and strong states of the SPV. The most commonly studied events are sudden stratospheric warmings (SSWs, Baldwin et al. (2021)), characterising the abrupt weakening of the SPV. SSW events are commonly defined by the reversal of the SPV mean flow from westerly to easterly (Charlton and Polvani, 2007; Butler et al., 2017; Palmeiro et al., 2015). In some studies, where the primary focus is on the abrupt dynamical nature of SSW events, a definition based on wind change is used (Birner and Albers, 2017; de la Cámara et al., 2019). On the contrary, events where the SPV becomes anomalously strong, with the mean flow accelerating to anomalously strong westerly values beyond a certain threshold, are characterised as strong vortex events (Tripathi et al., 2015). Due to the rapid nature of wave forcing, vortex weakening can be abrupt, whereas since vortex strengthening is driven by radiative processes, the strengthening is more gradual (Limpasuvan et al., 2005). The more rapid nature and stronger magnitude of vortex weakening than strengthening can be observed by comparing the magnitude of the identified vortex weakening and strengthening events in studies for SPV variability (e.g. Baldwin and Dunkerton, 2001; Limpasuvan et al., 2005). The asymmetry is also observed in the wave activity preceding the events (Polvani and Waugh, 2004) due to the strong relationship between wave forcing and mean flow.

Weak and strong states of the SPV can have a downward impact on surface weather that can last for a few weeks to a few months (Baldwin and Dunkerton, 2001). This downward influence can potentially be used to extend the predictability limit of surface weather from stratospheric origins (Domeisen et al., 2020a). In the stratosphere itself, the deterministic predictability limit of SSW events is about 10 days (Domeisen et al., 2020b; Taguchi, 2020), and it is found that the predictability of SSWs differs strongly between events (e.g. Karpechko, 2018). The source of predictability of SSW events is attributed in some studies to the predictability of wave activity (Stan and Straus, 2009; Karpechko et al., 2018) and tropospheric blocking (e.g. Tripathi et al., 2016), as blocking events often precede SSW events (e.g. Martius et al., 2009). It is found in ensemble forecasting systems that when the forecasts are initialised under strong blocking conditions, ensemble members of the forecasts 
can undergo bifurcation and lead to large uncertainties (Karpechko, 2018; Lee et al., 2019). However, even when successfully predicting a preceding blocking event, a model may still fail to predict a SSW (Tripathi et al., 2016), suggesting that another important factor for a successful predictions of SSWs is to accurately predict the initial stratospheric conditions.

Extreme stratospheric events, e.g. SSW and strong vortex events, are often the main focus of stratospheric predictability studies (e.g. Domeisen et al., 2020b; Taguchi, 2014, 2020). Strong vortex events are shown to be more predictable than SSW events (Domeisen et al., 2020b). To our knowledge, the reason for the observed differences in predictability between event types is, however, not resolved in existing literature, and is often attributed to the different mechanisms driving these events. The sample size of SSW and strong vortex events in sub-seasonal prediction systems tends to be too small to systematically assess their differences in predictability. Thus, in this study, we expand the analysis of the predictability of extreme stratospheric events to wind deceleration and acceleration events. As SSW events and strong vortex events are periods of strong zonal wind deceleration and acceleration, respectively, a better understanding of the predictability of wind deceleration and acceleration events will also contribute to the understanding of the predictability of SSW and strong vortex events. We aim to address the following questions: 1 . If we expand the event definitions to wind deceleration and acceleration events, do we also see a difference in predictability between wind deceleration and acceleration events, as for SSW and strong vortex events? 2. If so, what contributes to the difference in predictability between events? For example, is predictability related to event magnitude or event mechanisms? 3. What are the dynamical precursors for the predictability of the events? Do those precursors set the predictability limit of the events?

The paper is structured as follows: Section 2 discusses the data and methods adopted in this study. Section 3.1 illustrates the predictability differences between wind acceleration and deceleration events, Section 3.2 discusses the predictability dependence of events on event magnitude, and Section 3.3 explores the predictability dependence on event mechanisms. Finally, we discuss our results in Section 4.

\section{Data and methods}

\subsection{Datasets}

The hindcasts (retrospective forecasts) of the European Centre for Medium-Range Weather Forecasts (ECMWF) model from the subseasonal-to-seasonal (S2S) prediction database (Vitart et al., 2017) are used to evaluate the predictability of stratospheric events in Northern Hemisphere winter, from November to March (NDJFM), in the period of 1998/99-2017/18, which is the full available hindcast period for the model versions used in this study. The hindcasts are initialised twice a week (every Monday and Thursday) for the 20 year period alongside the real-time operational forecasts. The hindcasts consist of 11 ensemble members.

The model versions CY43R3 and CY45R1, corresponding to hindcasts with model version dates of 2017-07-13 to 201906-10, are used. Similar model configurations are used in both model versions used here, and they both use the ECMWF ERA-Interim reanalysis (Dee et al., 2011) for initialisation. The different model versions lead to qualitatively similar results in 
terms of prediction skill in their hindcasts (not shown) and are thus both used for the analysis presented here. The hindcasts are verified against the ERA-Interim reanalysis.

We evaluate the skill of the hindcasts at various lead times. Lead time is referred to as the time between the event onset date and the hindcast initialisation date. For example, a lead time of -5 indicates a hindcast initialised 5 days before the event onset. Hindcasts are divided into 6 lead time groups (LTGs) according to their initialisation dates, each of which represents a 5-day lead time window. For example, LTG-30 refers to hindcasts with initialisation dates of 30 to 26 days before the event, while LTG-5 refers to hindcasts from 5 days to 1 day before the onset date.

\subsection{Skill measures}

The following metrics are used to assess the predictability of stratospheric events: Mean error, continuous ranked probability score (CRPS), hit-rate (HR), and ensemble spread. The definitions are stated below.

1. Mean error

The mean error is the average difference between the hindcast $(F)$ and the observation $(O)$ (here, reanalysis is used instead of observations as the verification dataset). The index $i$ denotes the corresponding ensemble member, and $\mathrm{N}$ denotes the ensemble size. For the ECMWF model, $\mathrm{N}=11$. The perfect score of the mean error is 0.

Mean Error $=\frac{1}{N} \sum_{i=1}^{N}\left(F_{i}-O_{i}\right)$

2. Continuous ranked probability score (CRPS)

The CRPS measures the difference between the predicted cumulative distribution function $(\mathrm{CDF})\left(P_{f}(x)\right)$ of a variable $x$ and the observed $\mathrm{CDF}\left(P_{o}(x)\right)$. For ensemble forecasts, the predicted CDF is given by the predictions of all the ensemble members. The perfect score of the CRPS is 0 .

$C R P S=\int_{-\infty}^{\infty}\left(P_{f}(x)-P_{o}(x)\right)^{2} d x$

3. Hit-rate (HR)

The hit-rate (HR) is defined as the fraction of ensemble members that successfully predict an event, given by dividing the number of successful members $(M)$ by the total number of ensemble members $(N)$. A successful prediction requires that the model predicts an event of the same magnitude category as identified from reanalysis, i.e. a strong or weak magnitude event, on the same date as the event in reanalysis. The perfect score of the HR is 1. 


\section{Ensemble spread}

The ensemble spread of the ensemble members in a hindcast is measured as the standard deviation of the ensemble member predictions around the ensemble mean $(\bar{F})$. If the ensemble members show perfect agreement with each other, the ensemble spread is 0 .

$$
\text { Ensemble Spread }=\sqrt{\left[\frac{1}{N} \sum_{i=1}^{N}\left(F_{i}-\bar{F}\right)\right]^{2}}
$$

\subsection{Definition of stratospheric events}

From the daily mean of the zonal mean zonal wind at $60^{\circ} \mathrm{N}$ and $10 \mathrm{hPa}(\bar{u})$ from NDJFM 1998/99-2017/18 of ERA-Interim, we identify zonal wind acceleration and deceleration events. Both acceleration and deceleration events are defined as 10-day events and are identified using a 10-day moving window. Another event can only be identified 20 days after the start of an event to prevent identifying the same event. If a stronger deceleration is observed within 20 days of the last identified event, the period with stronger wind deceleration is selected instead, replacing the weaker event. The start date of the event is defined as day 0 of the event, i.e. the day when acceleration or deceleration starts in the 10-day window. The magnitude of the identified events is defined as the wind change over the 10-day event window, i.e. $\Delta \bar{u}=\bar{u}(t=9)-\bar{u}(t=0)$, where $t$ indicates the lead time. A 10-day event window is chosen as a result of a systematic comparison of different window widths (not shown). Although different processes are involved in deceleration and acceleration events, the duration of wind deceleration and acceleration is found to be similar (Fig. A1a). The event magnitude capture by a 10-day window also show reasonable values and not wind fluctuations (Fig. A1b). Therefore, also for comparability between the event types, we use the same event window width of 10 days to identify both wind deceleration and acceleration events.

The identified events are classified into weak and strong magnitude events. Events that have an absolute magnitude above the respective 60th percentile of the identified acceleration and deceleration events are classified as strong magnitude events, and those below are classified as weak magnitude events. The 60th percentiles are $16.94 \mathrm{~ms}^{-1}$ and $-24.55 \mathrm{~ms}^{-1}$ for the acceleration and deceleration events, respectively, in the reanalysis. In the ECMWF model, the 60th percentiles of the identified events are $16.77 \mathrm{~ms}^{-1}$ and $-20.87 \mathrm{~ms}^{-1}$ for the acceleration and deceleration events, respectively. The thresholds used here are comparable to the thresholds to define strong deceleration events used in other studies (e.g. Birner and Albers, 2017; de la Cámara et al., 2019).

For the acceleration and deceleration events identified from reanalysis, we check if they are also associated with extreme stratospheric events, i.e. SSWs, strong vortex events and vortex recovery events. SSW events are defined using the Charlton and Polvani (2007) wind reversal criterion. The onset date of an SSW event is identified as the first day that the daily mean zonal mean zonal winds at $60^{\circ} \mathrm{N} 10 \mathrm{hPa}$ are negative. The winds have to be westerly for at least 20 consecutive days before the event and return to westerly for at least 10 days after the event. We classify a deceleration event to be associated with an SSW event if an SSW occurs within the 10-day event window. The identified deceleration events can also be associated with early 
Table 1. Identified acceleration and deceleration events from reanalysis. The numbers in the brackets specify the number of events in each category.

\begin{tabular}{lccc}
\hline Acceleration event & Weak (51) & Strong (34) & Total (85) \\
\hline Strong vortex & 14 & 11 & 25 \\
\hline Vortex recovery & 8 & 11 & 19 \\
\hline Other acceleration events & 29 & 12 & 41 \\
\hline \hline Deceleration event & Weak (39) & Strong (26) & Total (65) \\
\hline SSW & 0 & 10 & 10 \\
\hline Other deceleration events & 39 & 16 & 55 \\
\hline
\end{tabular}

final warming (FW) events. Early FW events are defined as in Butler and Domeisen (2021) as those that occur at least 2 days before the median climatological FW date, which is Apr 12 over the period 1979-2019 in JRA-55 reanalysis. Since we only identify events up to March, the number of events associated with final warming events is small, and wave forcing still plays a dominant role in the FW wind reversal. Therefore, we keep the events associated with final warmings in the analysis and do not distinguish them from other deceleration events.

A strong vortex event is defined when $\bar{u}$ exceeds a threshold value. Following Tripathi et al. (2015) and Domeisen et al. (2020b), the chosen threshold value is $41.2 \mathrm{~m} / \mathrm{s}$, which is the 80th percentile of the zonal mean zonal wind averaged from November to March over the 1980-2012 period in ERA-Interim. We classify an acceleration event to be associated with a strong vortex event if the wind at any time during the event window is above this threshold. If the wind at $60^{\circ} \mathrm{N}, 10 \mathrm{hPa}$ at any time during the acceleration event window shows negative wind values, the event is classified as being associated with a vortex recovery event, which occur after SSW events. Table 1 shows the identified events from the reanalysis and their respective event types.

\subsection{Dynamical indices and significance tests}

As mentioned in the Introduction, we can quantify the preconditioning of the vortex background state, which guides waves towards the vortex, by the refractive index. As the refractive index is proportional to the meridional PV gradient $\left(\bar{q}_{y}\right)$ divided by the zonal mean zonal wind, following Jucker and Reichler (2018) and Albers and Birner (2014), we approximate the refractive index using the meridional PV gradient. Using the formulation of Equation (5) in Simpson et al. (2009), we divide 
the meridional PV gradient in spherical coordinates $\left(\bar{q}_{\phi}\right)$ by the radius of Earth $(a)$ to obtain an equation of the meridional PV gradient in Cartesian coordinates $\left(\bar{q}_{y}\right)$,

$\bar{q}_{y}=\frac{\bar{q}_{\phi}}{a}=\frac{2 \Omega \cos (\phi)}{a}-\left[\frac{(\bar{u} \cos \phi)_{\phi}}{a^{2} \cos \phi}\right]_{\phi}+\frac{f^{2}}{R_{d}}\left(\frac{p \theta}{T} \frac{\overline{u_{p}}}{\overline{\theta_{p}}}\right)_{p}$

where $\phi$ is the latitude, overline denotes the zonal mean, subscripts denote derivatives. As the term associated with Earth's rotation (first term in the equation) is small in extratropical and polar latitudes, and as the third term in the equation correlates well with the second term (not shown), we use the second term in Equation (5), $-\left[\frac{(\bar{u} \cos \phi)_{\phi}}{a^{2} \cos \phi}\right]_{\phi}$, as a proxy for waveguidability, hereafter referred to as $\bar{u}_{y y}$. Other than being a reasonable indicator for the refractive index, $\bar{u}_{y y}$ is a measure of the sharpness the edge of the stratospheric polar vortex, thus also a measure of the strength of the initial vortex state. Similar to Jucker and Reichler (2018), who used a polar cap averaged meridional PV gradient, we take a latitudinal average of $\bar{u}_{y y}$ over $55-75^{\circ} \mathrm{N}$ at $10 \mathrm{hPa}$. As a measure of upward wave activity in the lower stratosphere, following Polvani and Waugh (2004), we use the latitudinal average of meridional eddy heat fluxes $\left(\overline{v^{\prime} T^{\prime}}\right)$ over $45-75^{\circ} \mathrm{N}$ at $100 \mathrm{hPa}$, where $v$ is the meridional wind, $T$ is the temperature, and prime $\left({ }^{\prime}\right)$ denotes the departure from the zonal mean.

We use a one-sample t-test to assess the significance for the mean of a distribution. When comparing the significant difference between two distributions, we use a Kolmogorov-Smirnov test (KS test). For both tests, we use a confidence level of $95 \%$.

\section{Results}

\subsection{Predictability of stratospheric events in Northern Hemispheric winter}

To illustrate the predictability differences between stratospheric events, we compare the skill of the model in predicting different event types as a function of lead time. The magnitude of the events identified in reanalysis $(\Delta \bar{u})$, measured by the wind difference between day 9 and day 0 , predicted by the model hindcasts is compared against the same value in reanalysis for all lead time groups (Fig. 1). The left y-axis (blue) in Fig. 1 shows the errors in event magnitude for the deceleration and SSW events (as a subset of deceleration events). The right axis (red), which is flipped, shows the errors in event magnitude for the acceleration events and strong vortex events (as a subset of acceleration events). Values above the zero line indicate an underestimation of the magnitude of both deceleration and acceleration events, while values below zero indicate an overestimation. The box plots in Fig. 1 of most LTGs lie above zero, indicating an underestimation of event magnitude for both acceleration and deceleration events, including strong vortex events and SSWs. The underestimation of the event magnitude reduces towards smaller LTGs. At LTG-5, the model overestimates around 25\% of deceleration and 5\% of acceleration events, respectively, shown by the bottom of the box and whisker crossing the zero line. The underestimation of deceleration event magnitude is also seen in Karpechko (2018), where the model shows an initial weakening of the vortex but underestimates the event magnitude.

Previous studies that assessed the predictability of events using event onset dates have found that SSW events are less predictable than strong vortex events (e.g. Domeisen et al. (2020b)). This result is confirmed in Fig. 1: The mean errors for SSW 


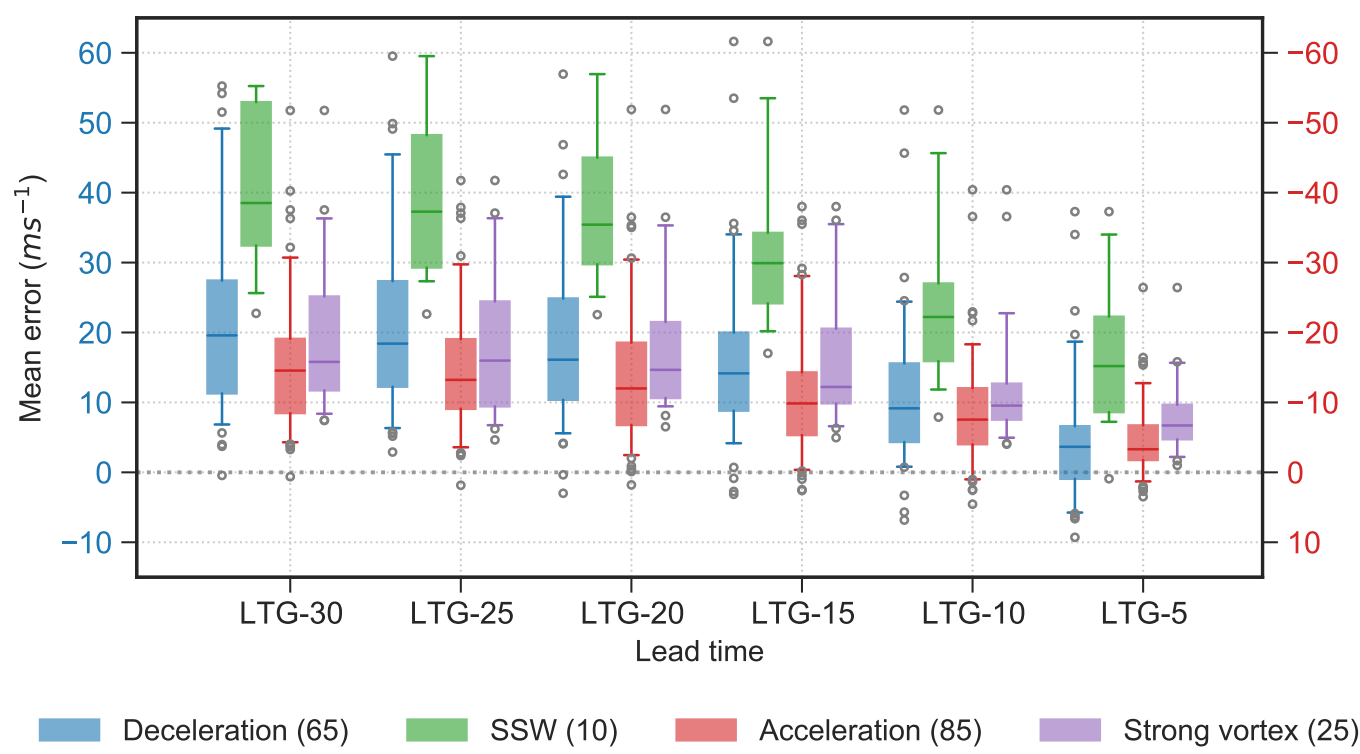

Figure 1. Mean error in the magnitude $(\Delta \bar{u})$ of deceleration events (blue), SSW events (green), acceleration events (red) and strong vortex events (purple), for all LTGs. For deceleration (blue) and SSW events (green), refer to the left axis (blue). For acceleration (red) and strong vortex events (purple), refer to the right axis (red). The box extends from the 25th to the 75th percentiles of the mean error of the events, with a horizontal line at the median. The whiskers extend from the 5th to the 95th percentiles. Outliers are plotted as grey open circles. The numbers in brackets correspond to the number of events in total for each event type in reanalysis.

events are larger than for strong vortex events, showing that SSW events are less predictable. Extending the analysis to wind deceleration and acceleration events, we also find that deceleration events are associated with larger errors than acceleration events at all lead times longer than 5 days. At LTG-5, the median of the mean error of deceleration events is lower than for acceleration events, but the distribution of mean errors (the extent of the box plot) remains larger than for the acceleration events, indicating that a greater uncertainty in predicting wind deceleration events remains in the model at short lead times.

\subsection{Predictability dependence on event magnitude}

To better understand the nature of the stratospheric events, we plot the distribution of the events identified from reanalysis (transparent bars in Fig. 2, which are the same in all panels). The events identified from reanalysis show an asymmetry in event magnitude, that is, deceleration events are associated with stronger magnitude than acceleration events. The median magnitude of reanalysis deceleration and acceleration events is $-21.25 \mathrm{~ms}^{-1}$ and $15.32 \mathrm{~ms}^{-1}$, respectively, and $-37.22 \mathrm{~ms}^{-1}$ and $15.06 \mathrm{~ms}^{-1}$, respectively, for SSW events and strong vortex events. All SSW events belong to the strong deceleration events category, whereas the magnitudes of the strong vortex events are spread more evenly across the weak and strong acceleration event categories (Table 1). The stronger magnitude of deceleration events as compared to acceleration events is consistent 
with Limpasuvan et al. (2005), i.e. that the daily zonal mean zonal wind anomalies observed for vortex weakening events are stronger than for vortex strengthening events.
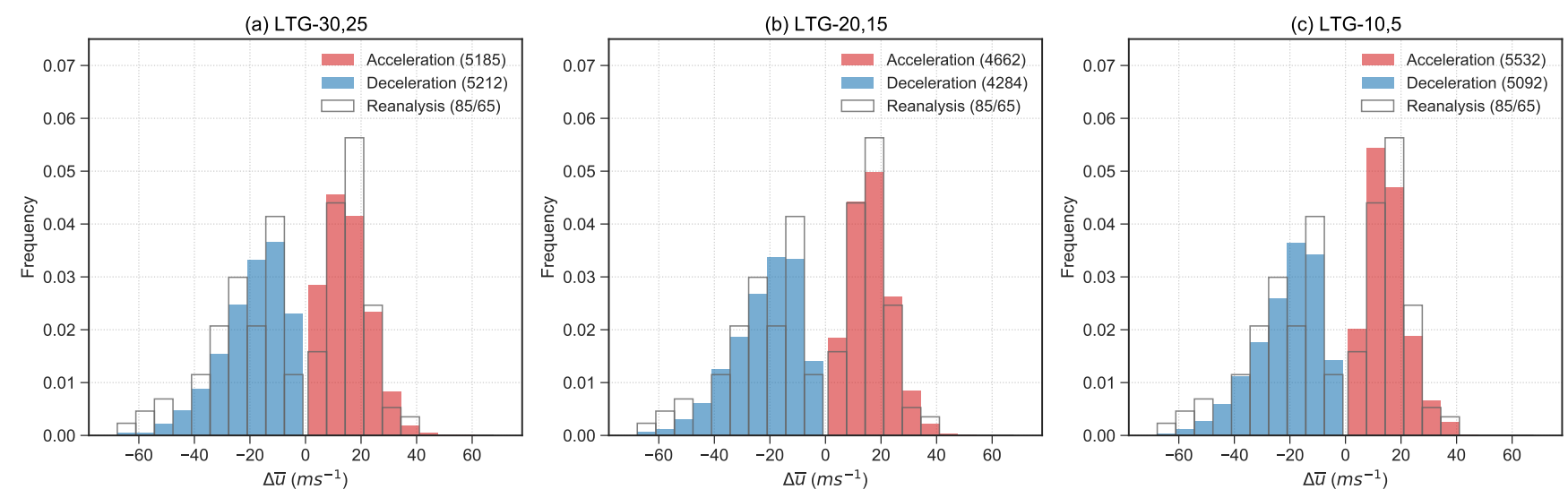

Figure 2. Distributions of the wind change $(\Delta \bar{u})$ of the acceleration (red) and deceleration (blue) events identified from reanalysis (transparent bars with grey outline) and from the ensemble members in the hindcasts that are initialised in NH winter at (a) LTG-30,25, (b) LTG-20,15 and (c) LTG-10,5. Numbers in brackets indicate the number of identified events at each lead time. The reanalysis distributions displayed in all panels are identical and the numbers in brackets refer to the number of acceleration / deceleration events. The histograms are normalised.

As deceleration events have a stronger magnitude than acceleration events and as the identified events span a wide range of magnitudes, as a first step, we test if the differences in predictability between events arise from different event magnitudes. We plot the CRPS of the model in predicting the event magnitude against the observed event magnitude at different lead times (Fig. 3). The grey diagonal line in each panel indicates when a hindcast exhibits a skill equal to a climatological prediction, as the CRPS is the difference between the predicted and observed distribution, the CRPS given by validating the predicted climatological event magnitude against the observed event magnitude will equal to the observed event magnitude itself, which will lie on the diagonal line. Thus, points above the diagonal line show a poorer skill than a climatological prediction, and the points below show a skill that is improved with respect to climatology. The closer the points are to the $x$-axis, i.e. the line of CRPS $=0$, the more skilful the hindcasts.

For long lead times of around 30 days, the fitted lines lie just below the diagonal line (Fig. 3a), which suggests that the hindcasts exhibit a predictability that is just slightly better than climatological forecasts at these lead times. The fitted slopes then approach the x-axis with decreasing lead time (going from panels (a) to (f)), indicating that, as expected, the model gains more information from initial conditions and the prediction is improved beyond climatological values. The predictability behaviour of both acceleration and deceleration events can roughly be approximated by a linear fit, indicating that the stronger the event magnitude, the less predictable the event. The linear fits corresponding to the deceleration and acceleration events overlap within the $95 \%$ confidence interval (blue and red shading, respectively) at all lead times, suggesting that the acceleration and deceleration events show the same predictability behaviour. 

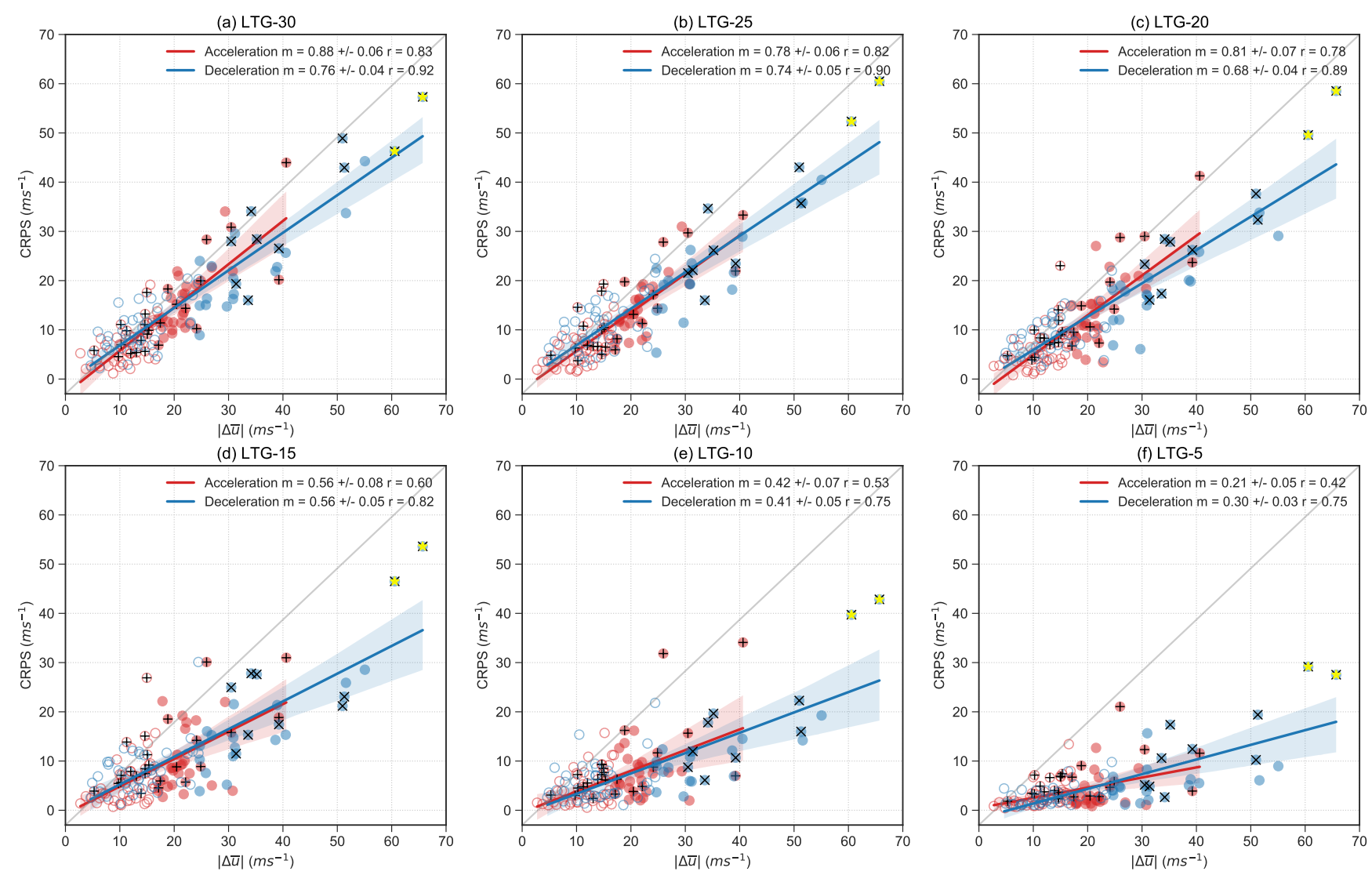

Figure 3. CRPS of event magnitude $(\Delta \bar{u})$ for the identified wind acceleration (red) and deceleration (blue) events plotted against their absolute event magnitude $(|\Delta \bar{u}|)$ from reanalysis for different LTGs. The absolute value of event magnitude $(|\Delta \bar{u}|)$ is used for a better comparison between acceleration and deceleration events. The filled circles represent strong magnitude events and the empty circles represent weak magnitude events. Linear regression lines are fitted to each of the LTGs, $m$ indicates the slope, including the standard error of the fit. Pearson correlation coefficients ( $\mathrm{r}$ ) are indicated in the legend for acceleration and deceleration events, respectively, and $\mathrm{r}$ is statistically significant at $95 \%$ for all panels. The shaded region shows the $95 \%$ confidence interval of the linear fit. Pluses ('+') indicate events that correspond to strong vortex events and crosses ('X') correspond to SSW events. Yellow stars ('*') denote the 2009 and 2018 split SSW events.

At short lead times, most of the points lie close to zero CRPS. Some events, however, retain a large CRPS and deviate from the linear fit in the direction of the diagonal line. For instance, the two extreme SSW events with magnitudes of over $60 \mathrm{~ms}^{-1}$ (marked with yellow stars in Fig. 3). The fact that the CRPS remains larger for the two events at LTG-5 suggests that the model might not be capturing the precursors or that it might not accurately represent the mechanisms required to predict these events.

To better illustrate the predictability dependence on event magnitude, we composite the strong and weak magnitude events, i.e. events with magnitudes above and below the 60th percentile, respectively, and compare their averaged skill at different 

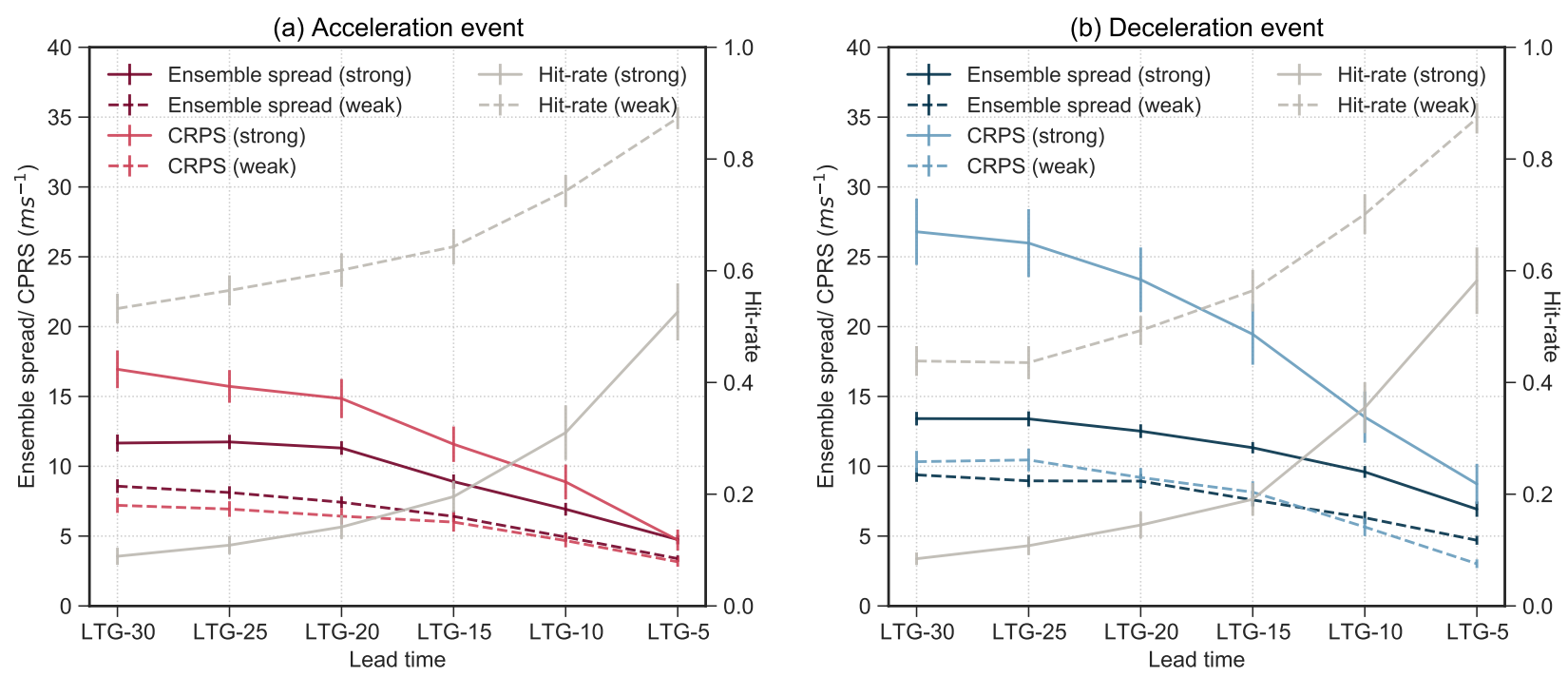

Figure 4. Ensemble spread (dark colour), CRPS (light colour) and hit-rate (grey) for (a) acceleration events (red) and (b) deceleration events (blue) computed by validating the hindcasts against the reanalysis. Solid lines indicate the mean of the strong magnitude events at different LTGs and the dotted lines indicate weak magnitude events. The vertical bars indicate the standard errors for each LTG.

lead times (Fig. 4). Overall, as expected from the model capturing more of the required precursors to predict the events, both acceleration and deceleration events show an increase in hit-rate, and a decrease in ensemble spread and CRPS with decreasing lead time. Strong magnitude events exhibit poorer skill than weak magnitude events, associated with a lower hit-rate, and a larger ensemble spread and CRPS. As large ensemble spread can be observed in ensemble forecasting systems when the forecast is initialised under e.g. strong blocking conditions (Lee et al., 2019; Karpechko, 2018), this might indicate that strong magnitude events are associated with strong precursors or forcings that are not as well captured by the model as those for weak magnitude events. We will discuss the predictability dependence on event mechanism in Section 3.3.

\subsection{Predictability dependence on event mechanism}

In the last section, we showed that event magnitude strongly determines the predictability, with strong events being less predictable, which can be described mostly by a linear behaviour. Some events, however, deviate from this behaviour, which might be connected the mechanism of the events. In this section, we investigate whether the background state of the SPV and the drivers to the events can have an influence on the predictability of events. We start this section by linking the predictability of the events to the related mechanisms through both the influence of the background state of the stratosphere and the drivers in terms of the upward wave flux for both the reanalysis and the prediction system. 
(a) Deceleration events $\bar{u}_{y y}$

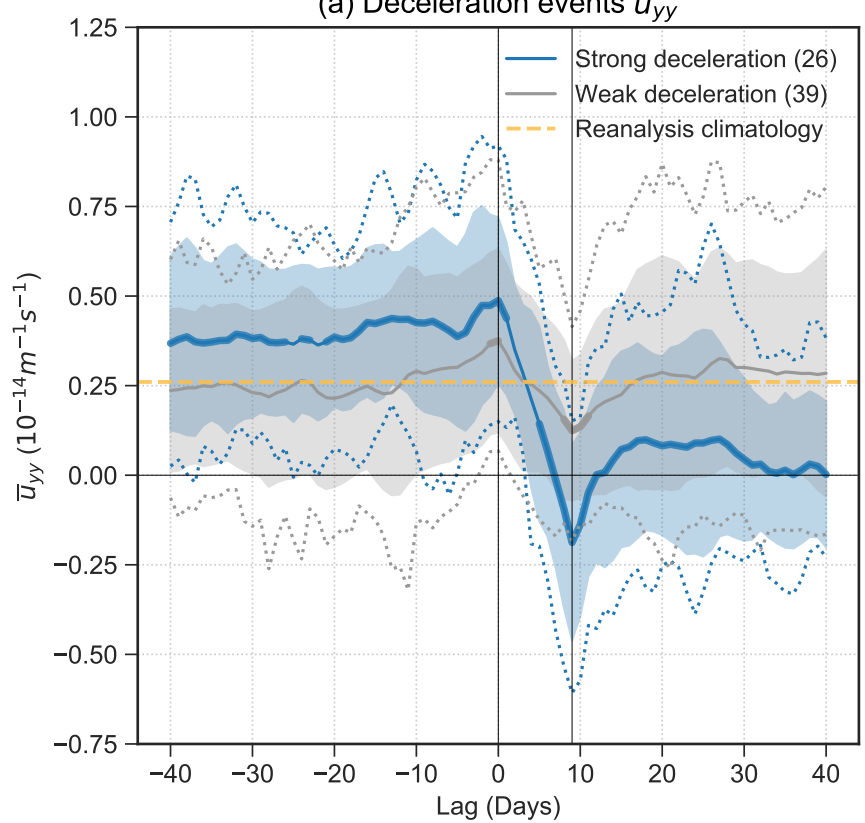

(c) Acceleration events $\bar{u}_{y y}$

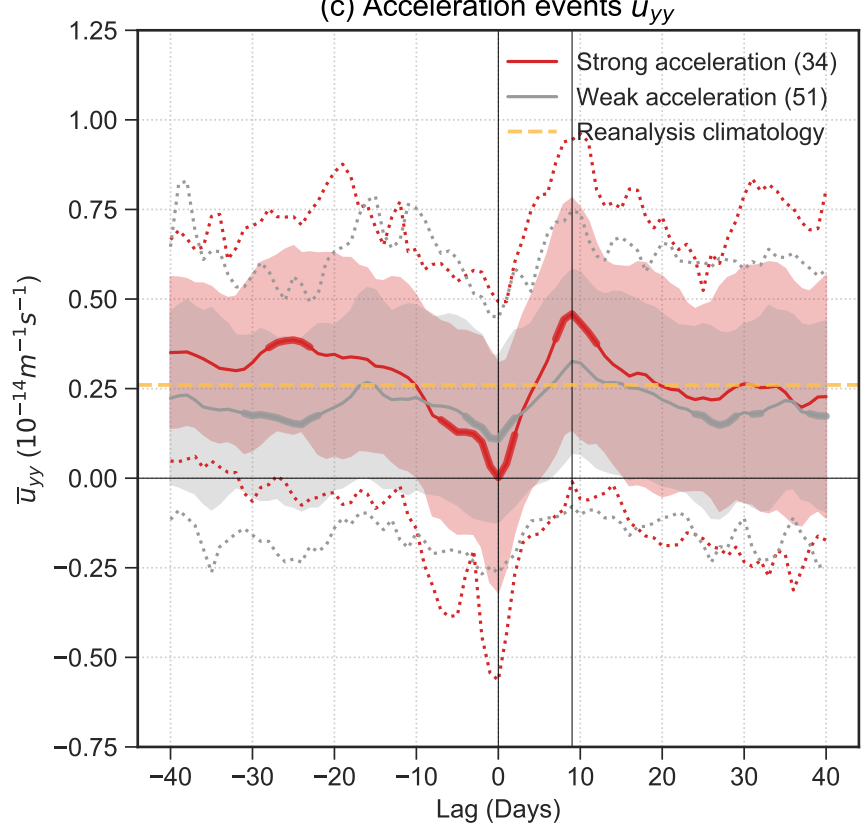

(b) Deceleration events $\overline{V^{\prime} T^{\prime}}$

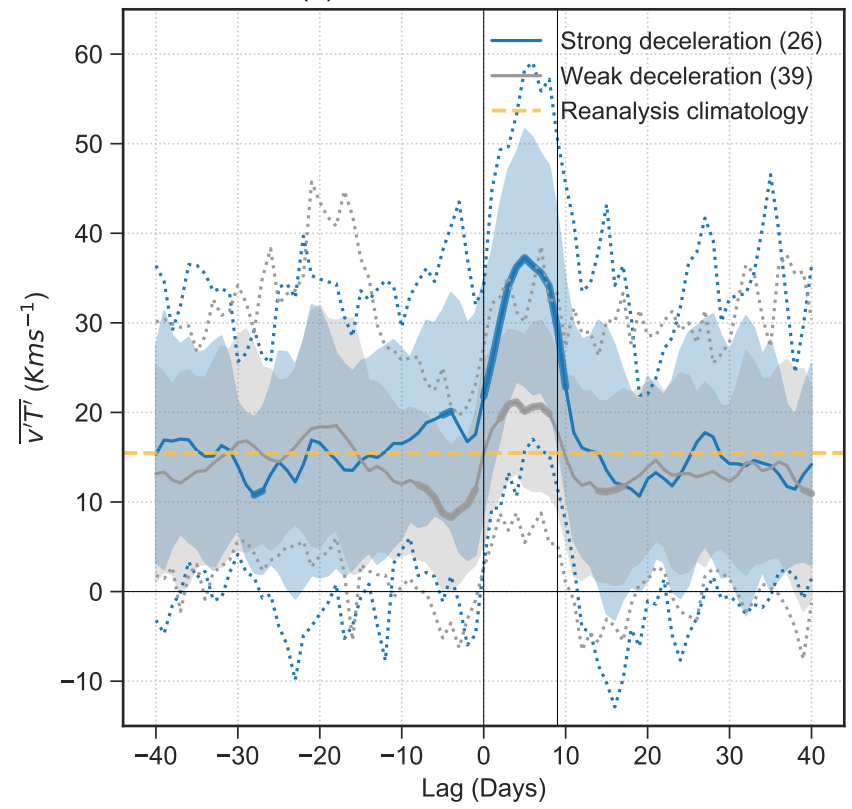

(d) Acceleration events $\overline{v^{\prime} T^{\prime}}$

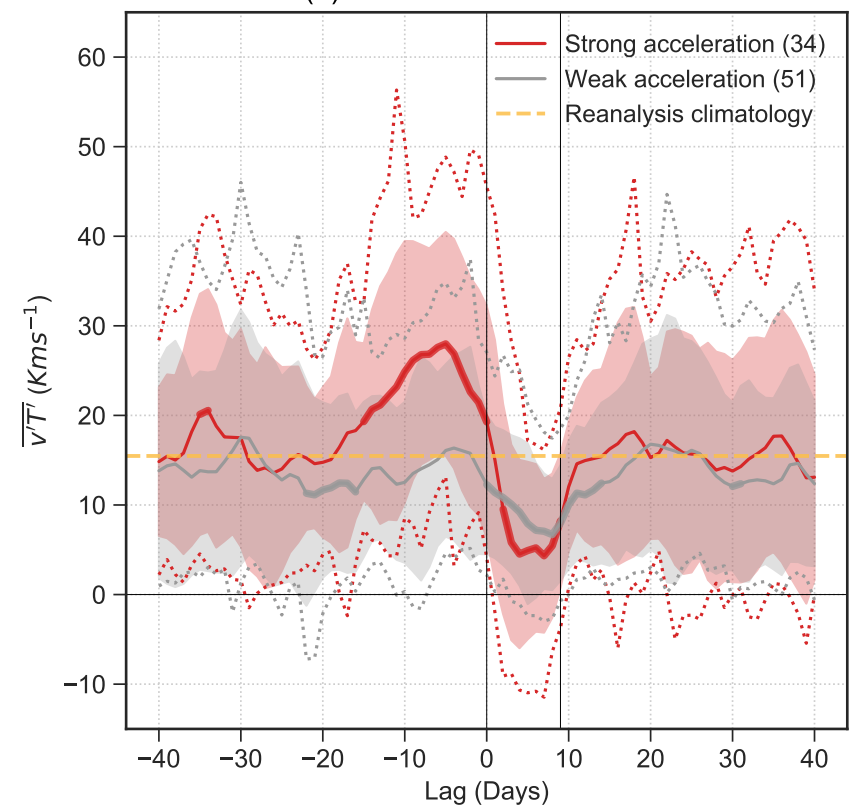

Figure 5. Time evolution of daily values of $\bar{u}_{y y}(\mathrm{a}, \mathrm{c})$ and $\overline{v^{\prime} T^{\prime}}(\mathrm{b}, \mathrm{d})$ for the strong deceleration (blue) (a, b) and acceleration (red) (c, d) events in reanalysis. The solid line is the mean value of all events and the bold parts of the line indicate lags where the composites are significantly different from the reanalysis winter climatology (dotted yellow lines) at 95\% using a student's t-test. Weak events are composited separately and shown in grey. The dotted lines in the corresponding colours indicate the 5th and 95th percentiles of the composite, the shaded regions indicate the 25 th to 75 th percentiles. The numbers in the brackets of the legend indicate the number of events in each composite. Lag is relative to the first day of the identified 10-day events. 
https://doi.org/10.5194/wcd-2021-84

Preprint. Discussion started: 11 January 2022

(c) Author(s) 2022. CC BY 4.0 License.

(c) (i)

Weather and

Climate Dynamics

Discussions

\subsubsection{Vortex background state in reanalysis}

Before strong deceleration events, $\bar{u}_{y y}$ is significantly stronger than climatology (Fig. 5a), confirming the existing literature that preconditioning of the vortex via sharpening of the vortex edge is often observed before weak vortex events (e.g. Limpasuvan et al., 2004; Jucker and Reichler, 2018). During strong deceleration events (days 0 to 9), $\bar{u}_{y y}$ reduces to a negative value that is significantly weaker than climatology. The vortex recovers at the end of the strong deceleration event and the mean value of $\bar{u}_{y y}$ returns to positive values, but is still significantly weaker than climatology up to 40 days after the event onset. For weak deceleration events, the values of $\bar{u}_{y y}$ before and after the events are close to climatology, and significant signals are only found during the event window (day 0 to 9), suggesting that the preconditioning of the vortex background state before event onset might not be as important for weak deceleration events. For strong acceleration events, increased $\bar{u}_{y y}$ is found only at around 25 days before the events and a few days later, $\bar{u}_{y y}$ decreases to values significantly lower than climatology (Fig. 5c). During strong acceleration events, $\bar{u}_{y y}$ increases to a value significantly above climatology and drifts back to climatology after the event. For weak acceleration events, a few periods of anomalously weak $\bar{u}_{y y}$ are observed at around day -25, 0, 25.

To further illustrate the relationship between $\bar{u}_{y y}$ and event magnitude $(\Delta \bar{u})$, we plot $\Delta \bar{u}$ against $\bar{u}_{y y}$ at day 0 for all events (Fig. 6a,d). A significant negative correlation is found between $\bar{u}_{y y}$ and $\Delta \bar{u}$ for deceleration events, that is, the stronger $\bar{u}_{y y}$, the stronger the deceleration. For the acceleration events, a weak but significant negative correlation is also found for $\bar{u}_{y y}$ on day 0 against $\Delta \bar{u}$ (Fig. $6 \mathrm{~d}$ ). But as the wind change for acceleration events has the opposite sign of deceleration events, the negative correlation shows that the weaker the $\bar{u}_{y y}$, the stronger the wind acceleration.

\subsubsection{Wave activity forcing in reanalysis}

In addition to the background state, the forcing by drivers is responsible for extreme stratospheric events. In particular, anomalous wave activity in the lower stratosphere drives the deceleration of the SPV mean flow (Polvani and Waugh, 2004; Hinssen and Ambaum, 2010). The 10-day event window captures well the onset of wind deceleration (Fig. A2a) and the anomalously strong wave activity during the event (Fig. 5b). The wave activity starts to increase from day 0, peaks around day 5 and then decreases to a value that is not significantly different from climatology at the end of the event on day 9 . As expected, the wave activity is much stronger during the strong deceleration events than during the weak deceleration events. We find a significant negative correlation when correlating the integrated sum of $\overline{v^{\prime} T^{\prime}}$ during the event window (day 0 to 9) with the deceleration event magnitude $(\Delta \bar{u})$ (Fig. 6c). The stronger the wave activity during the event window, the more the wind decelerates. For our definition of deceleration events, using a 10-day event window, SSWs occur on average around day 9 of the event window. Therefore, the peak of $\overline{v^{\prime} T^{\prime}}$ during the event window is consistent with our understanding that anomalous wave activity precedes SSW events (e.g. Butler et al. (2017)). A wave activity lower than climatology is found around 10 days before (day -10 to -1) the weak magnitude deceleration events but not the strong magnitude events, suggesting the occurrence of weak acceleration events before weak deceleration events. Wind acceleration is indeed observed 10 days before the weak deceleration events, and the magnitude of the acceleration is similar to the magnitude of the subsequent deceleration events (Fig. A2a). Plotting the integrated $\overline{v^{\prime} T^{\prime}}$ for days -10 to -1 against $\Delta \bar{u}$ during the event window, we observe a weak negative correlation 

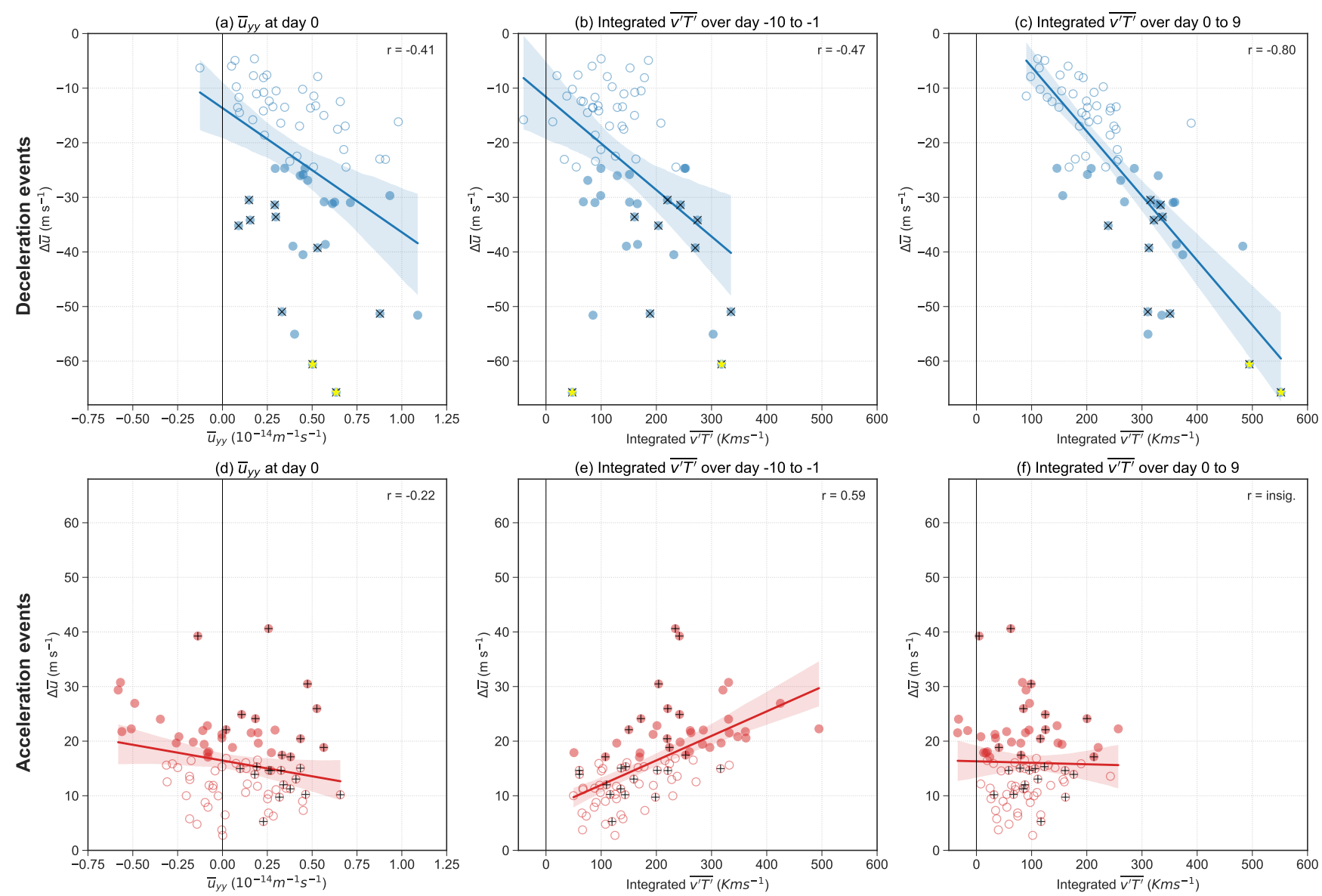

Figure 6. Relationship between the magnitude of the deceleration events $(\Delta \bar{u})$ and (a) $\bar{u}_{y y}$ at day 0 , (b) integrated $\overline{v^{\prime} T^{\prime}}$ over day -10 to -1 and (c) integrated $\overline{v^{\prime} T^{\prime}}$ over day 0 to 9. (d),(e) and (f) same as (a-c) but for the acceleration events. Filled (empty) circles indicate strong (weak) magnitude events, ' $\times$ ' indicates SSW events and '+' indicates strong vortex events. The solid line indicates the fitted linear regression line and shading indicates the $95 \%$ confidence interval. Pearson correlation coefficients $(r)$ are significant at $95 \%$ in all panels. Yellow stars ('*') in (a-c) denote the 2009 and 2018 split SSW events.

(Fig. 6b), which can be explained by low wave activity preceding the weak deceleration events and slightly increased wave activity preceding the strong deceleration events.

Weaker than climatological wave activity $\overline{v^{\prime} T^{\prime}}$ is observed during the acceleration event window (Fig. 5d). The wave activity is similar for strong and weak acceleration events but slightly lower for the strong acceleration events. Although strong acceleration events are associated with lower wave activity, there is no significant relationship between the integrated heat flux and event magnitude (Fig. 5f), indicating that wave activity does not drive the acceleration event magnitude, and low wave activity might be more of a threshold criterion for an acceleration event to occur. The passive role of wave activity in wind acceleration is consistent with our understanding that radiative cooling drives the wind acceleration when the wave activity 
is below a critical level. Interestingly, we observe strong heat flux from around 15 days before the strong acceleration events (Fig. 5d). The same is observed when we exclude vortex recovery events in the composite (not shown). We find a significant positive correlation between the integrated $\overline{v^{\prime} T^{\prime}}$ for days -10 to -1 and the wind change over the acceleration event window (day 0 to 9) (Fig. 6e). We find that deceleration events precede about $74 \%$ of the strong acceleration events (not shown). The deceleration events that happen before the acceleration events can weaken the vortex, preconditioning the background state of the vortex to be more favourable for the onset of acceleration events, consistent with the weakening of $\bar{u}_{y y}$ before the onset of strong acceleration events from around day -10 (Fig. 5c). The alternation between deceleration and acceleration events is reminiscent of the characteristics of stratospheric vacillations as described in the Holton-Mass model (Holton and Mass, 1976), which shows an oscillation of the mean flow of the vortex after an initial wave forcing.

It is interesting to note that the two strongest strong vortex events, the events with a magnitude of around $40 \mathrm{~ms}^{-1}$, are further away from the linear fit, suggesting that factors other than low wave activity might play a role for these strong magnitude events, for example, strong ozone depletion (e.g., Haase and Matthes, 2019; Lin et al., 2017).

\subsubsection{Representation of dynamical processes in the model}

Deceleration and acceleration events are found to be driven by anomalies in $\bar{u}_{y y}$ and $\overline{v^{\prime} T^{\prime}}$ as described in Section 3.3.1 and 3.3.2 using reanalysis. We now assess the ability of the model to represent these anomalies and relationships. We start by treating all ensemble members independently and identify deceleration and acceleration events from each separate member at different lead times.

Overall, the climatology of the model event magnitude is similar to that observed in reanalysis at all lead times (Fig. 2). Using a KS test for the model distributions for acceleration and deceleration events, respectively, against the reanalysis distributions, the model and reanalysis distributions are found to not be significantly different from each other. A similar number of events is identified at all lead times, but slightly fewer at LTG-20,15. At LTG-30,25, the model shows an overall underestimation of event magnitude and produces more events with a magnitude close to zero in the model as compared to reanalysis, which is consistent with the predictions being close to climatology at long lead times (Fig. 3a,b). The number of events with very weak magnitude decreases when events are identified at shorter lead times. At all lead times, the model underestimates the number of extremely strong deceleration events (shown by the difference between the model and reanalysis in the negative tails of the distributions). The model covers the range of acceleration event magnitude well but underestimates the frequency of acceleration events with moderate magnitude, i.e. around magnitudes of $20 \mathrm{~ms}^{-1}$ over the 10-day event window.

To assess the ability of the model in representing the event mechanisms, we composite the identified strong magnitude events from the model and compare the model evolution of the dynamical variables to the observed evolution in reanalysis (Fig. 7). The model shows a time evolution of $\bar{u}$ similar to that from reanalysis. However, as the model underestimates the number of deceleration events with extremely strong magnitude (Fig. 2), the mean evolution of $\bar{u}$ for deceleration events at all lead times in the model stays above zero, while the winds in reanalysis cross the zero wind line (Fig. 7a). The 5th and 95th percentiles of the model events (shadings) shifted towards more positive $\bar{u}$ as compared to reanalysis (dotted lines) around the end of the event window, indicating that the model does not reach values of $\bar{u}$ that are as small as observed in reanalysis. The mean 

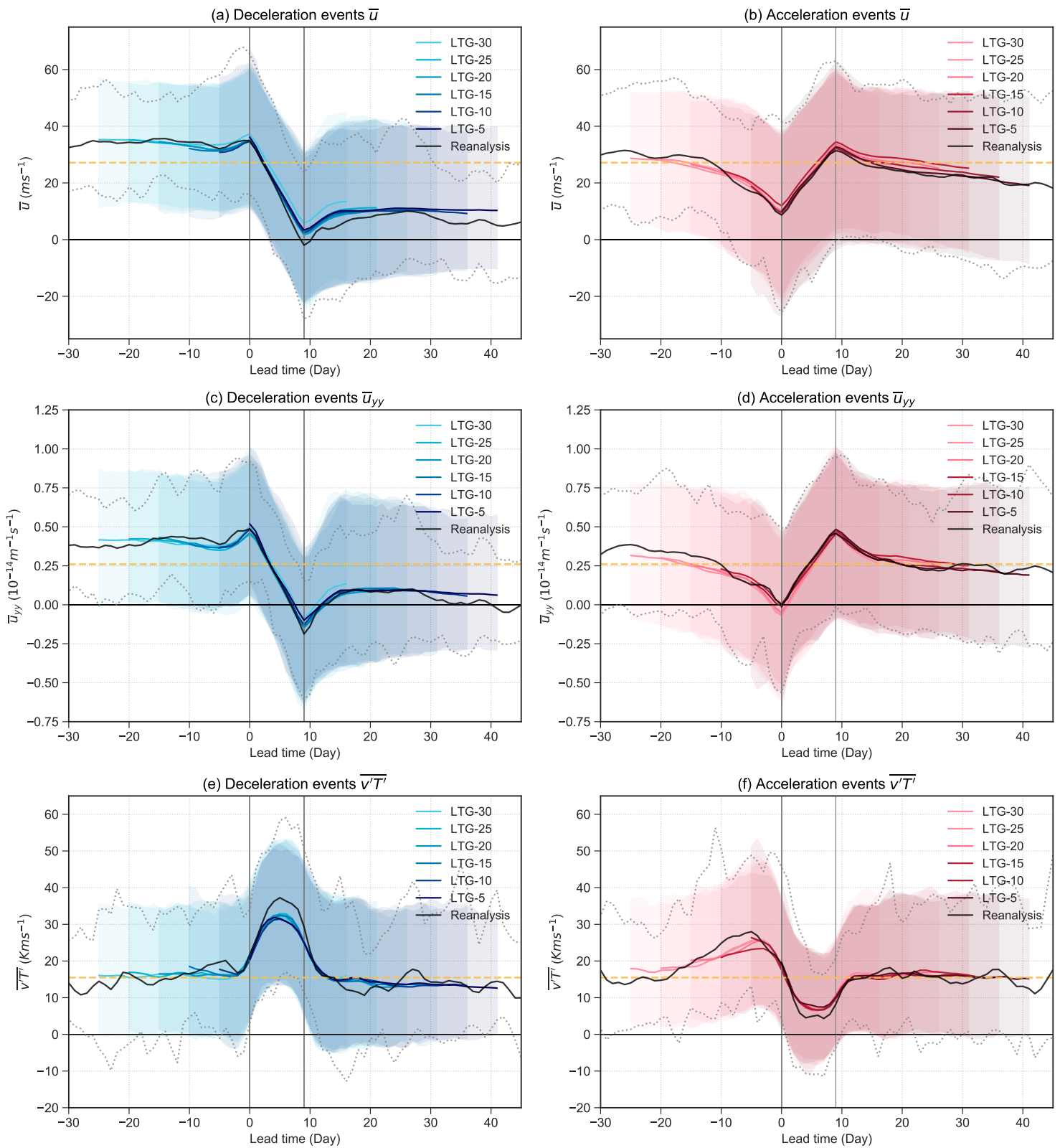

Figure 7. Temporal evolution of $\bar{u}, \bar{u}_{y y}$, and $\overline{v^{\prime} T^{\prime}}$ for (a),(c),(e) the strong deceleration and (b),(d),(f) the strong acceleration events identified in the model at different LTGs. Solid lines indicate the mean of the event composites and shadings indicate the 5th and 95th percentiles of the events in the prediction system. Black solid lines and black dotted lines indicate the mean, 5th and 95th percentiles for the reanalysis, respectively. Yellow dotted lines show the winter climatology in reanalysis. The first and last 5 days in the mean evolution in the model composite are discarded to account for the different start dates within each LTG. 
evolution of $\bar{u}$ at LTG-30 (lightest blue) remains above the values for all other LTGs throughout the event window until the end of the forecast.

The vortex background state is well represented in the model at all lead times for both strong deceleration and acceleration events. The model shows near identical mean values and variability comparable to the reanalysis for events identified at all lead times (Fig. 7c,d). For the wave forcing (Fig. 7e,f), the model events do not show the extremely high values of $\overline{v^{\prime} T^{\prime}}$ during strong deceleration events, or the extremely low values of $\overline{v^{\prime} T^{\prime}}$ during strong acceleration events, at all lead times. The 95th percentile of $\overline{v^{\prime} T^{\prime}}$ for the deceleration events in reanalysis is outside of the 95th percentile of the model (colour shadings). Similarly, for the acceleration events, the 5th percentile of the reanalysis composite is outside that of the model. Before acceleration events, a peak of $\overline{v^{\prime} T^{\prime}}$ is also observed in the model. However, the wave activity in the model for this peak before acceleration events peaks at a later time and at a lower magnitude.

Given the strong relationship observed between event magnitude and wave activity for deceleration events in reanalysis (Fig. 6c), the observed underestimation of strong $\overline{v^{\prime} T^{\prime}}$ for deceleration events in the prediction system might explain the observed underestimation of model deceleration event magnitude in Fig. 7a. As a sensitivity experiment, Fig. 7a and 7e are replotted by excluding the events with magnitude above the 90th percentile from the reanalysis composite of strong deceleration events (Fig. A3). It is found that the averaged evolution of $\bar{u}$ and $\overline{v^{\prime} T^{\prime}}$ of the model composite then covers almost the full variability of the re-computed reanalysis composite, and the evolution of the model composite is near identical to the reanalysis composite and covers almost the full range of the 5th and 95th percentiles. This suggests that the model has limitations in producing events that have equivalent event magnitudes of above the 90th percentile of the reanalysis deceleration events, likely due to not producing the required strong wave activity. The model also does not show as low $\overline{v^{\prime} T^{\prime}}$ during strong acceleration events (Fig. 7f). Although we see the model can produce acceleration events with an evolution similar to reanalysis (Fig. 7b), showing a good variability of acceleration event magnitude in the model, the frequency of acceleration events with moderate event magnitude might still be underestimated (as earlier discussed in Fig. 2). As discussed in Section 3.3.2, $\overline{v^{\prime} T^{\prime}}$ might be more a threshold criterion for acceleration events to occur. Specifically, if the wave activity produced in the model is not low enough in some occasions, this can contribute to an underestimation in the number of acceleration events with moderate magnitude, consistent with Figure 2.

We found that overall the model is able to produce events with a range of magnitude similar to reanalysis and has a good representation of event mechanisms. The model, however, has limitations in producing extremely strong anomalies in heat fluxes, thus might be underestimating the number of moderate magnitude acceleration events and the number and magnitude of extremely strong deceleration events. To elucidate the sources of predictability for the events, we now evaluate the magnitude of the anomalies in the precursors, i.e. in $\bar{u}_{y y}$ and $\overline{v^{\prime} T^{\prime}}$, captured by the model when predicting the events identified from reanalysis (Fig. 8).

At LTG-30,25, the anomalies in the precursors captured by the model are weak, indicated by the predicted distributions for both acceleration and deceleration events centering around the climatological values (Fig. 8e,i) which is reflected in the fact that acceleration and deceleration events at these lead times are barely separated (Fig. 8a). This is also consistent with Fig. 3 that the points lie close to the diagonal line at long lead times. Nevertheless, the predicted distributions of $\Delta \bar{u}$ are skewed towards the 

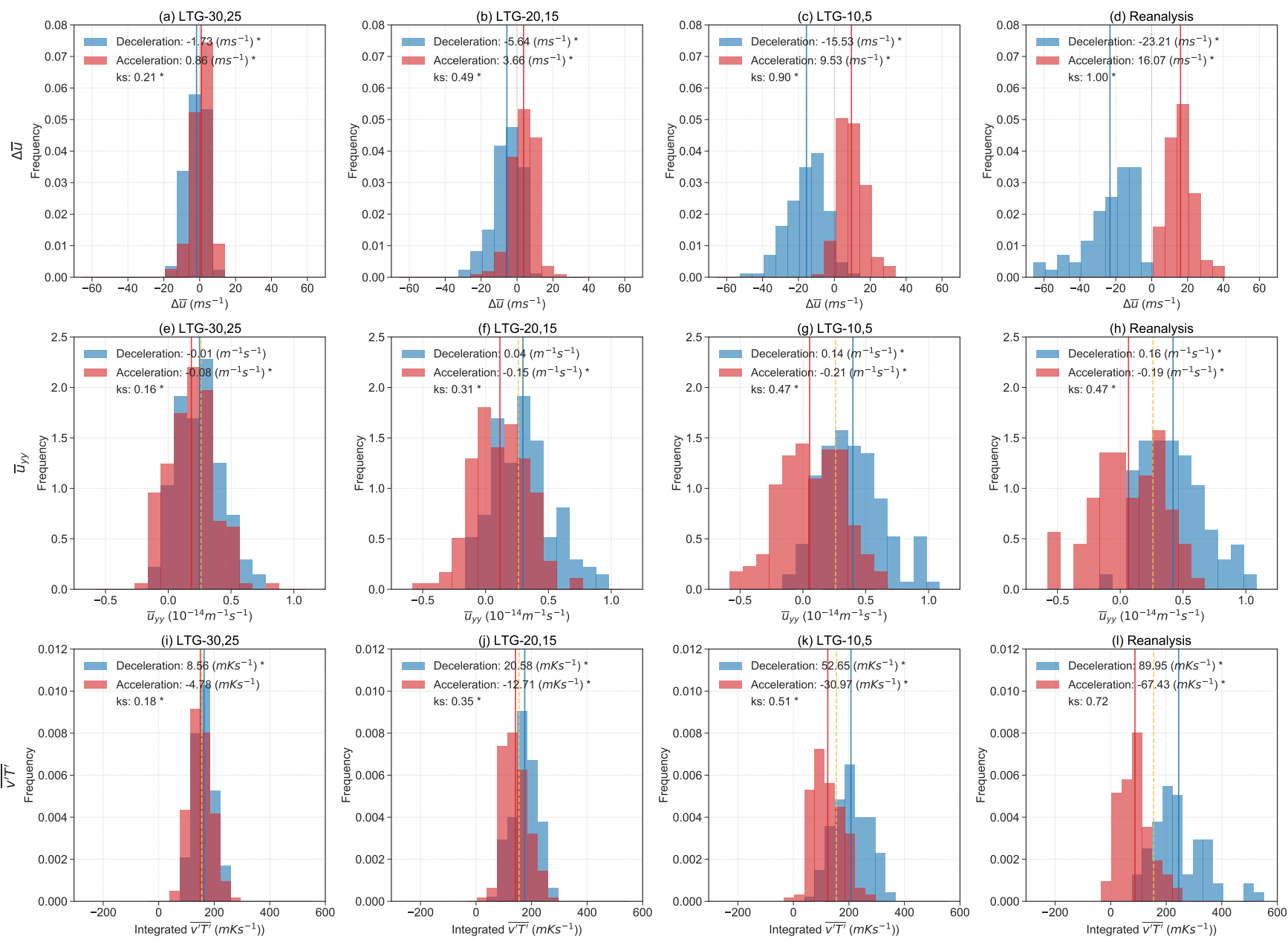

Figure 8. Ensemble mean values of $\Delta \bar{u}(\mathrm{a}-\mathrm{c}), \bar{u}_{y y}$ at day $0(\mathrm{e}-\mathrm{g})$ and integrated $\overline{v^{\prime} T^{\prime}}$ over day 0 to 9 (i-k) predicted by the model for the events diagnosed in reanalysis at different lead times. The observed distributions from reanalysis are shown in panels (d),(h) and (l). Deceleration events are shown in blue and acceleration events in red. The winter climatological values of $\bar{u}_{y y}$ and integrated $\overline{v^{\prime} T^{\prime}}$ from reanalysis are plotted as yellow dotted lines. The differences of the mean of the distributions from 0 (for $\Delta \bar{u}$ ) or from climatology (for $\bar{u}_{y y}$ and integrated $\left.\overline{v^{\prime} T^{\prime}}\right)$ are shown in the legend. * indicates when the distributions are significantly different from 0 in (a) to (d) and when the distributions differ from the reanalysis climatological value in (e) to (l) using a t-test. The histograms are normalised and a KS test is used to test the significant difference between the acceleration and deceleration event distributions. Statistically significant KS statistics $(k s)$ are indicated by $\mathrm{a}^{*}$.

correct signs of the observed events (e.g. the predicted wind change for deceleration events is skewed towards negative values) (Fig. 8a). The predicted distributions for the precursors of acceleration and deceleration events are significantly different from each other, for all lead times, showing that the magnitude of the precursors captured for acceleration and deceleration events are statistically distinguishable even at long lead times. 
For shorter lead times, the predicted distributions for acceleration and deceleration become more clearly distinct as the difference between the predicted deceleration and acceleration distribution increases (indicated by greater distance between the distribution means and higher KS test score at shorter lead times). However, comparing the predicted distributions to the observed distribution from reanalysis (the very right column), the model shows a clear underestimation of the very strong deceleration events (with $\Delta \bar{u}$ stronger than $-40 \mathrm{~ms}^{-1}$ ) and an underestimation in the very high values of integrated $\overline{v^{\prime} T^{\prime}}$. The frequency of values with around $400 \mathrm{mKs}^{-1}$ are underestimated and values above $400 \mathrm{mKs}^{-1}$ are scarcely predicted in the model (Fig. 8k,l). For acceleration events the distribution of the wind change even includes negative values in the predicted event magnitude distribution at LTG-10,5 (Fig. 8c), which is not the case in reanalysis (Figure 8d). As a result, the mean of the predicted $\Delta \bar{u}$ distribution is lower than for reanalysis. The model predicts more acceleration events with $\Delta \bar{u}$ close to $0 \mathrm{~ms}^{-1 /}$ 10 days and some acceleration events with negative $\Delta \bar{u}$. The model also shows a lower frequency of $\overline{v^{\prime} T^{\prime}}$ than the reanalysis (Fig. 8k and 81).

To quantify the contribution of the predictability of the precursors to the predictability of event magnitude at different lead times, we plot the CRPS of the event magnitude against the CRPS of the precursors (Fig. 9). A significant correlation is found between the CRPS of the event magnitude and of the precursors for both acceleration and deceleration events at all lead times. Consistent with Fig. 8, the model captures the anomalies of the precursors more accurately with decreasing lead time. Specifically, as the CRPS in $\bar{u}_{y y}$ and integrated $\overline{v^{\prime} T^{\prime}}$ decreases, the CRPS in $\Delta \bar{u}$ also decreases. On the other hand, the CRPS of $\Delta \bar{u}$ shows a stronger correlation with the CRPS of integrated $\overline{v^{\prime} T^{\prime}}$ than $\bar{u}_{y y}$, indicating a stronger contribution of the predictability of integrated $\overline{v^{\prime} T^{\prime}}$ to the predictability of event magnitude, which is consistent with the more direct role of $\overline{v^{\prime} T^{\prime}}$ than $\bar{u}_{y y}$ in forcing the events.

The largest CRPS values in $\overline{v^{\prime} T^{\prime}}$ are found for the 2009 and 2018 split SSW events (yellow stars in Fig. 9). These two SSW events were associated with very strong wave-2 activity (Ayarzagüena et al., 2011; Domeisen et al., 2018). The large $\overline{v^{\prime} T^{\prime}}$ for these events might be out of the range of $\overline{v^{\prime} T^{\prime}}$ that the model can produce, as suggested earlier, or the mechanisms for these events may not be properly represented in the model.

\section{Conclusions}

By expanding the stratospheric event definition to wind deceleration and acceleration events using the tendency of the zonal mean zonal wind at $60^{\circ} \mathrm{N}$ and $10 \mathrm{hPa}$, we systematically investigate the predictability of extreme events in the SPV in the ECMWF S2S hindcasts. We demonstrate that, overall, the ECMWF model represents the variability of the SPV well in terms of event magnitude and the associated dynamical drivers, and it has a good representation of the dynamical processes that are observed in reanalysis. The model, however, shows limitations in producing events with extremely strong deceleration magnitudes. We find that this is associated with the inability of the model to produce extremely strong wave activity in the lower stratosphere.

The large number of identified deceleration and acceleration events allows us to robustly compare the differences in the event mechanisms in both reanalysis and the model, and to understand the differences in the predictability between events. 


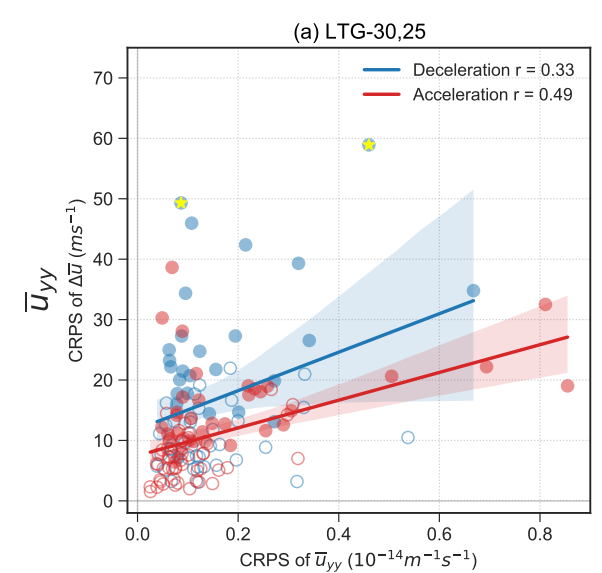

(d) LTG-30,25

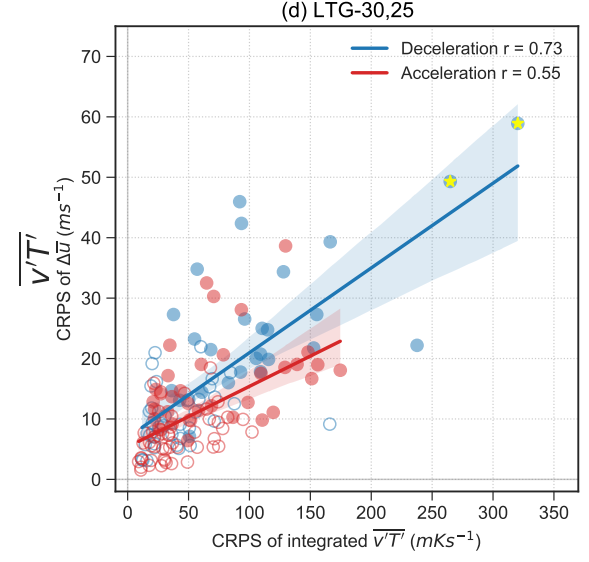

(b) LTG-20,15

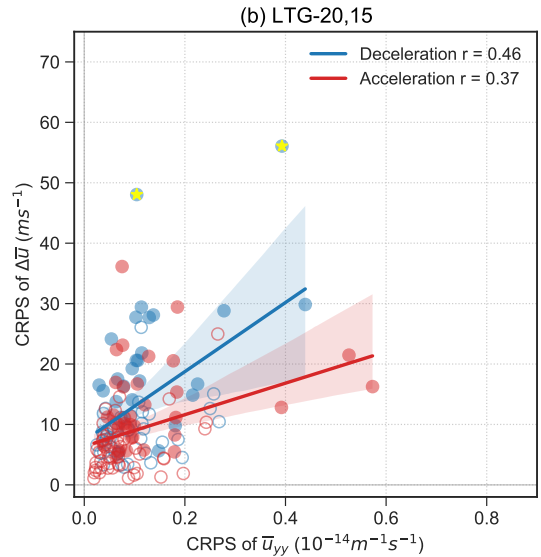

(e) LTG-20,15

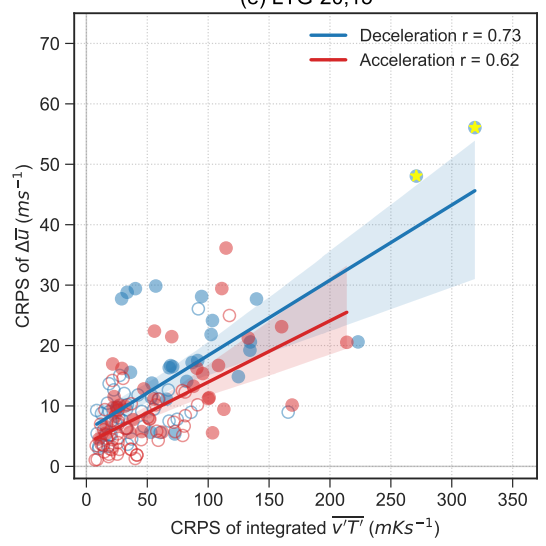

(c) LTG-10,5

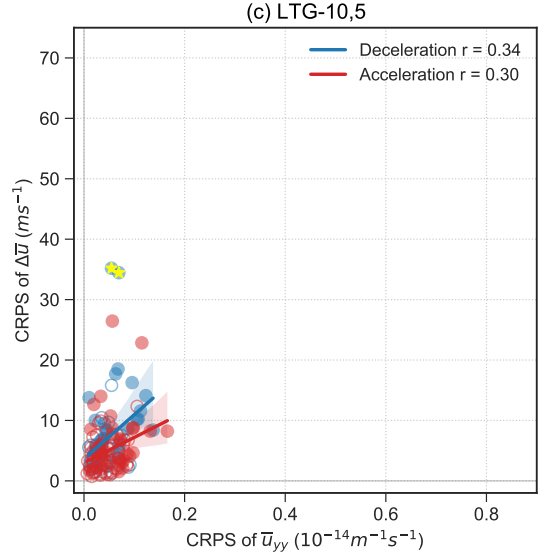

(f) LTG-10,5

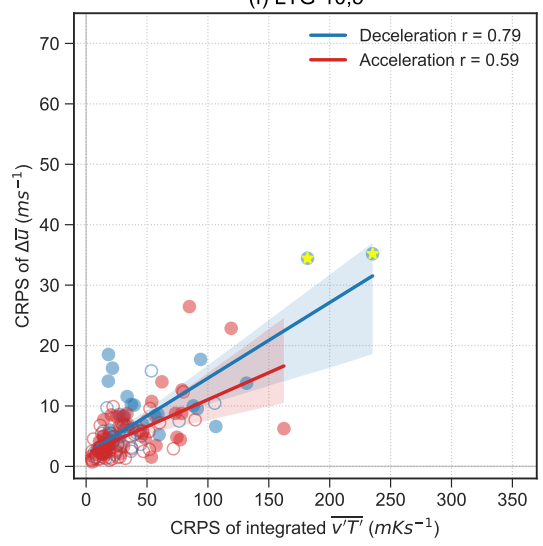

Figure 9. Relationship between the CRPS of $\Delta \bar{u}$ with (a-c) the CRPS of $\bar{u}_{y y}$ on day 0 and with (d-f) the CRPS of integrated $\overline{v^{\prime} T^{\prime}}$ over days 0 to 9 for the acceleration (red) and deceleration events (blue) identified from reanalysis. The solid line and the shading correspond to the fitted slope and $95 \%$ confidence interval of the fit. The Pearson correlation coefficients $(r)$ indicate the correlation in the scatter plots and are statistically significant in all panels at the 95\% level. Yellow stars ('*') denote the 2009 and 2018 split SSW events.

Consistent with our understanding of the mechanisms of wind deceleration and acceleration events in the framework of wavemean flow interaction, we find that deceleration and acceleration events are associated with the same anomalies but of opposite signs, namely a strengthened waveguide, in terms of the second meridional derivative of the zonal wind $\left(\bar{u}_{y y}\right)$, and higher wave activity for deceleration events, measured by the $100 \mathrm{hPa}$ eddy heat flux $\left(\overline{v^{\prime} T^{\prime}}\right)$, and vice versa for acceleration events. The predicted distributions of the acceleration and deceleration events become more distinct at shorter lead times and the respective characteristics of the distributions become better represented. For example, the long tails of deceleration events towards strong events become better represented, although the model continues to underestimate these long tails, even at short lead times.

A large part of the predictability differences between events can be explained by the different event magnitudes. When we express the predictability of deceleration and acceleration events in terms of event magnitude, we found that they both show a predictability dependence on event magnitude; that is, events of stronger magnitude are less predictable. We explain 
the observed predictability dependence from two perspectives: 1) In a statistical sense, strong magnitude events lie within the tails of the climatological distribution and are penalised more heavily than weak magnitude events, and 2) from a dynamical perspective, strong magnitude events are associated with strong anomalies in $\overline{v^{\prime} T^{\prime}}$ and $\bar{u}_{y y}$. The strong precursor anomalies are often less predictable in the model and thus can lead to large uncertainties in event magnitude. The same predictability behaviour with respect to event magnitude for deceleration and acceleration events thus suggests that the observed predictability difference between the event types can to a large extent be explained by the difference in event magnitude between the event types, i.e. the fact that wind deceleration events are associated with greater magnitudes than wind acceleration events, and that SSW events are stronger in magnitude than strong vortex events. We also show that the predictability of the $\overline{v^{\prime} T^{\prime}}$ and $\bar{u}_{y y}$ can explain most of the predictability of the events, with $\overline{v^{\prime} T^{\prime}}$ contributing a larger part of the predictability as compared to $\bar{u}_{y y}$. The predictability limit of these dynamical precursors might, therefore, set the predictability limit of events.

For a few events, large errors in the prediction remain even at short lead times, for example, the split SSW events in 2009 and 2018, which are the events with the two strongest event magnitudes of all deceleration and acceleration events investigated in this study. The two split events are reported to be associated with anomalously strong wave- 2 wave activity (Harada et al., 2010) and are also reported to be more unpredictable than other SSW events (Rao et al., 2018). The large errors associated with certain events even at short lead time suggest that these events might be associated with mechanisms different than weaker magnitude events. For example, internal stratospheric dynamics might play a more important role (e.g. Plumb, 1981; Matthewman and Esler, 2011; Domeisen et al., 2018), which might not be well represented in the model.

Further work is needed to understand the potential reasons as to why the model shows limitation in producing extremely strong wave activity. One might want to investigate whether the wave amplification mechanisms are different in the 2009 and 2018 split SSW events than other deceleration events, and to see whether the mechanisms associated with the two events are well represented in the model. A better representation of the wave amplification mechanisms and extremely strong wave activity in the model can potentially enhance the predictability of stratospheric events, and by extension their impacts on surface weather and climate.

\section{Appendix A}

To choose a suitable event window width for identifying acceleration and deceleration events, we study the variability of the SPV through the tendency in the zonal mean zonal wind at $60^{\circ} \mathrm{N}, 10 \mathrm{hPa}$ in reanalysis. We identify periods that show consecutive days of wind acceleration and deceleration by counting the number of consecutive days that the daily wind change is of the same sign. If the wind changes sign on one day, that day is counted as a new period of wind change. The number of days in the identified wind change period is defined as the duration.

The duration distribution for wind acceleration and deceleration is qualitatively similar to each other, both following an exponential distribution (Fig. A1a). The magnitude is given by the wind change over the duration of a given identified period. The duration and event magnitude shows a near relationship (Fig. A1b). 
https://doi.org/10.5194/wcd-2021-84

Preprint. Discussion started: 11 January 2022

(c) Author(s) 2022. CC BY 4.0 License.

(a) Duration

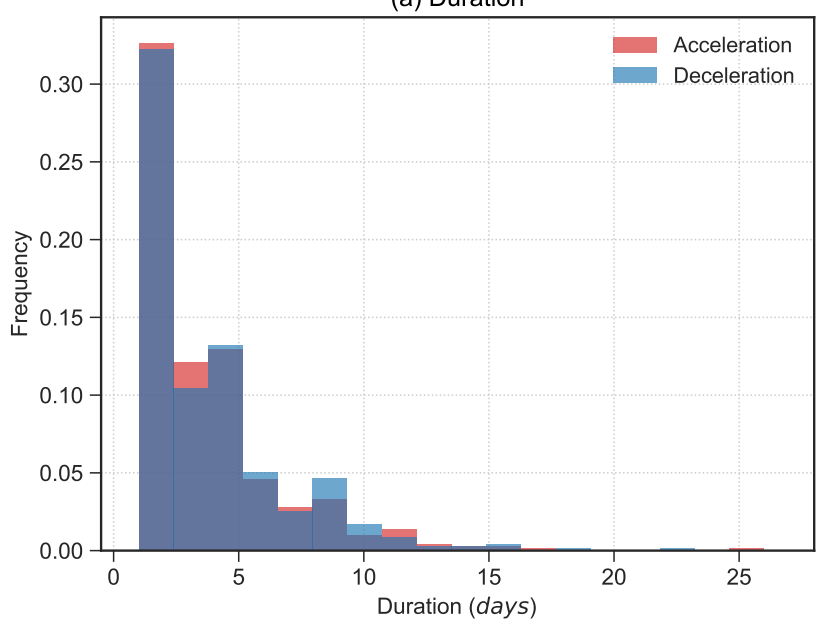

(b) Magnitude vs Duration

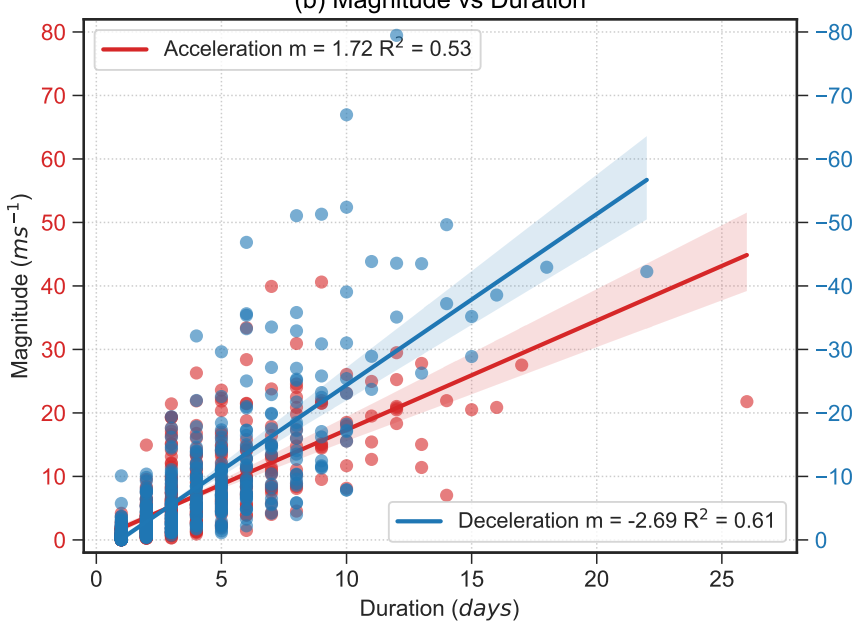

Figure A1. Periods of wind acceleration (red) and deceleration (blue) in reanalysis. (a) Duration, (b) the relationship between the magnitude and duration of the wind acceleration and deceleration periods. For acceleration periods (red), refer to the red axis on the left. For deceleration periods (blue), refer to the blue axis on the right. The solid lines mark the linear fit to the scatter plots and the shading marks the $95 \%$ confidence interval of the fit. The histograms are normalised. 
(a) Deceleration events $\bar{U}$

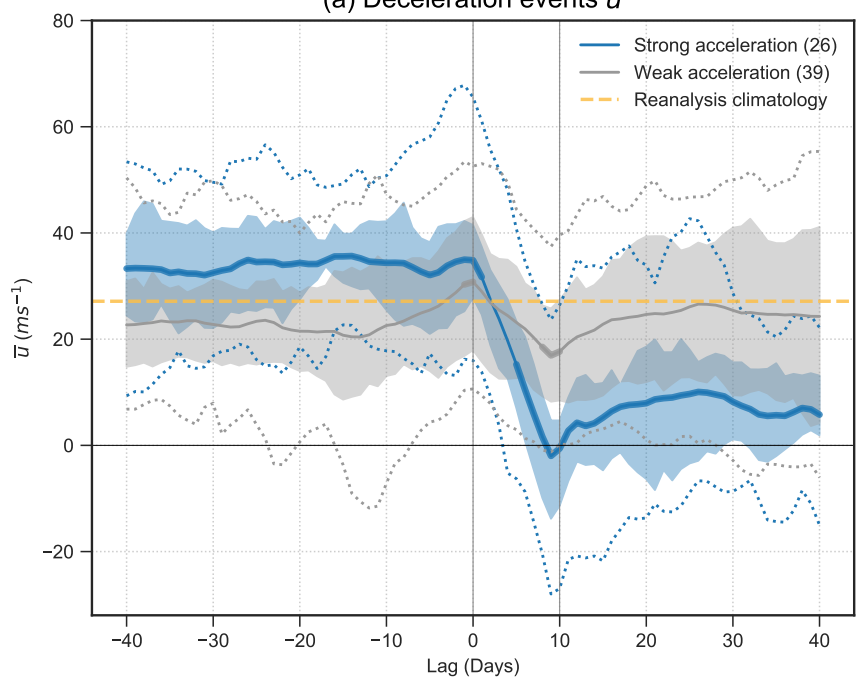

(b) Acceleration events $\bar{U}$

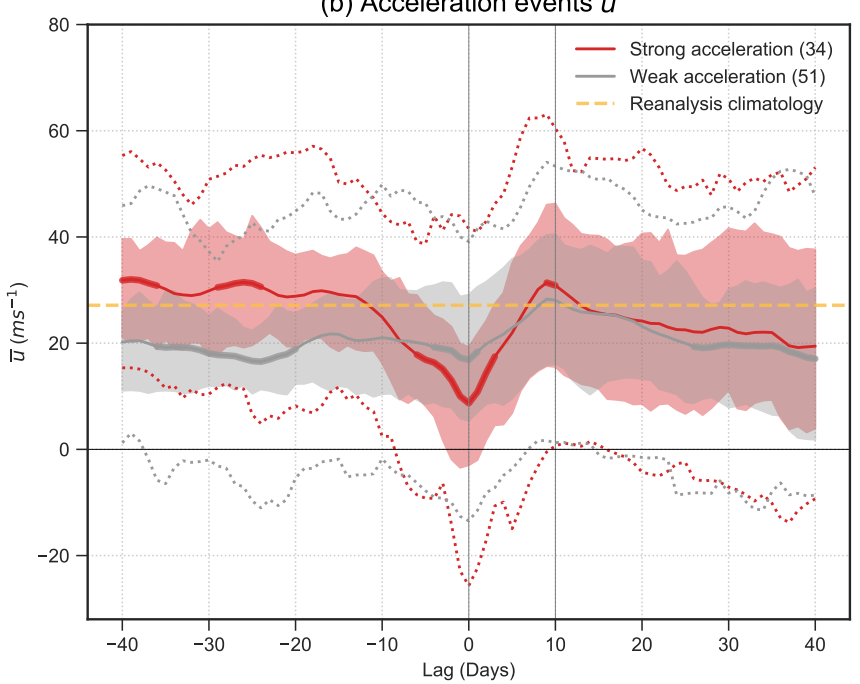

Figure A2. Time evolution of daily values of $\bar{u}$ for the strong deceleration (blue) and acceleration (red) events in reanalysis. The solid line shows the mean value and the bold line indicates where the composites are significantly different from the reanalysis climatology using a student's t-test. Weak events are composited separately and shown in grey. The dotted lines in the corresponding colours indicate the 5th and 95th percentile of the composite, the shaded region indicates the 25 th to 75 th percentiles. The dotted yellow line shows the winter climatology $\bar{u}$ in reanalysis. The number in the brackets of the legend indicates the number of events in the composites. Lag is relative to the first day of the identified 10-day events.

(a) Deceleration events $\bar{u}$

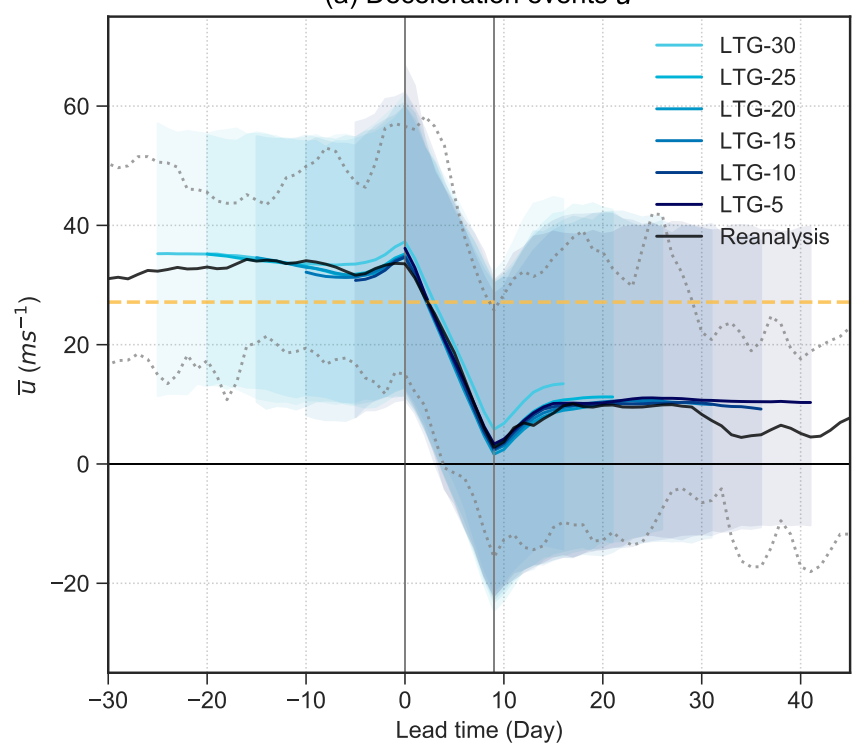

(b) Deceleration events $\overline{v^{\prime} T^{\prime}}$

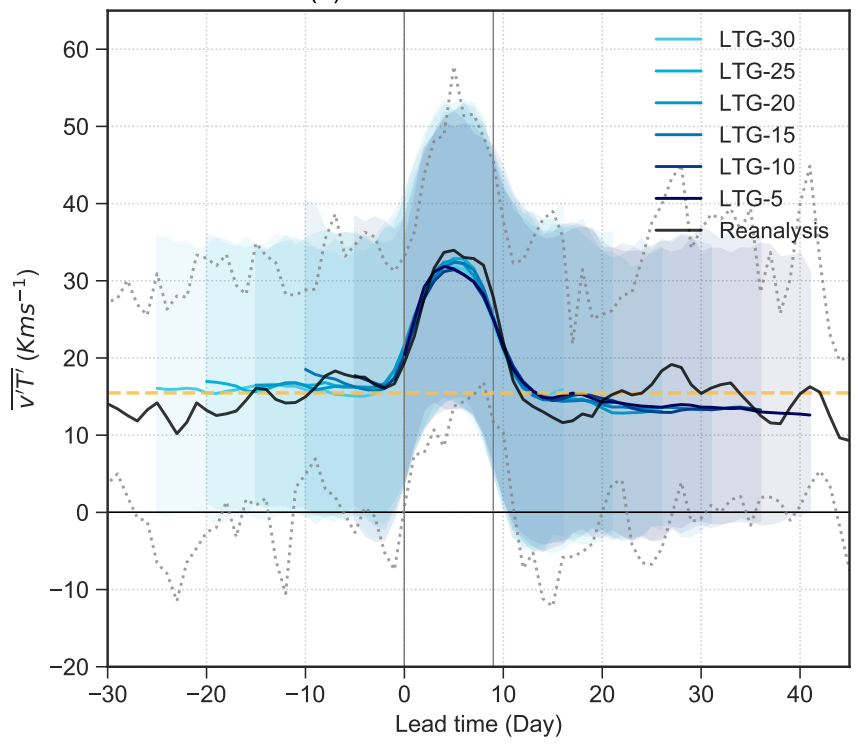

Figure A3. Like Figures 7a and 7e but excluding events with magnitude above the 90th percentile in the reanalysis composite. 
https://doi.org/10.5194/wcd-2021-84

Preprint. Discussion started: 11 January 2022

(c) Author(s) 2022. CC BY 4.0 License.

(c) (1)

Weather and

Climate Dynamics

Discussions

Author contributions. R.W. and D.D. designed the study. R.W. performed the analysis, made the figures, and wrote the manuscript draft. All authors discussed the research and worked on revising the manuscript.

Competing interests. The authors declare no competing interests.

Acknowledgements. The authors thank Huw Davies, Hilla Afargan-Gerstman, and Bernat Jiménez-Esteve for helpful discussions on earlier 440 versions of this research, and Ole Wulff for help with the S2S data. The work of R.W. is funded through ETH grant ETH-05 19-1 "How predictable are sudden stratospheric warming events?". The work of Z.W. is funded by the Swiss Data Science Center within the project EXPECT (C18-08). Support from the Swiss National Science Foundation through projects PP00P2_170523 and PP00P2_198896 to D.D. is gratefully acknowledged. The ERA-Interim data was obtained from https://apps.ecmwf.int/datasets/ and the S2S data was obtained from https://apps.ecmwf.int/datasets/data/s2s-reforecasts-instantaneous-accum-ecmf/levtype=pl/type=cf/. 
https://doi.org/10.5194/wcd-2021-84

Preprint. Discussion started: 11 January 2022

(c) Author(s) 2022. CC BY 4.0 License.
Weather and

Climate Dynamics

Discussions

\section{References}

Albers, J. R. and Birner, T.: Vortex preconditioning due to planetary and gravity waves prior to sudden stratospheric warmings, Journal of the Atmospheric Sciences, 71, 4028-4054, https://doi.org/10.1175/JAS-D-14-0026.1, 2014.

Ayarzagüena, B., Langematz, U., and Serrano, E.: Tropospheric forcing of the stratosphere: A comparative study of the two different major stratospheric warmings in 2009 and 2010, J. Geophys. Res. Atmos., 116, https://doi.org/10.1029/2010JD015023, 2011.

Baldwin, M. P. and Dunkerton, T. J.: Stratospheric Harbingers of Anomalous Weather Regimes, Science, 294, https://doi.org/10.1126/science.1063315, 2001.

Baldwin, M. P., Ayarzagüena, B., Birner, T., Butchart, N., Butler, A. H., Charlton-Perez, A. J., Domeisen, D. I. V., Garfinkel, C. I., Garny, H., Gerber, E. P., Hegglin, M. I., Langematz, U., and Pedatella, N. M.: Sudden Stratospheric Warmings, Rev. Geophys., 59, e2020RG000 708, https://doi.org/10.1029/2020RG000708, 2021.

Birner, T. and Albers, J. R.: Sudden Stratospheric Warmings and Anomalous Upward Wave Activity Flux, SOLA, 13A, 8-12, https://doi.org/10.2151/sola.13A-002, 2017.

Butler, A. H. and Domeisen, D. I. V.: The wave geometry of final stratospheric warming events, Weather Clim. Dyn., 2, 453-474, https://doi.org/10.5194/wcd-2-453-2021, 2021.

Butler, A. H., Sjoberg, J. P., Seidel, D. J., and Rosenlof, K. H.: A sudden stratospheric warming compendium, Earth Syst. Sci. Data, 9, 63-76, https://doi.org/10.5194/essd-9-63-2017, 2017.

Charlton, A. J. and Polvani, L. M.: A New Look at Stratospheric Sudden Warmings. Part I: Climatology and Modeling Benchmarks, Journal of Climate, 20, 449-469, 2007.

Charney, J. G. and Drazin, P. G.: Propagation of planetary-scale disturbances from the lower into the upper atmosphere, J. Geophys. Res., 66, 83-109, https://doi.org/10.1029/JZ066i001p00083, 1961.

de la Cámara, A., Birner, T., and Albers, J. R.: Are Sudden Stratospheric Warmings Preceded by Anomalous Tropospheric Wave Activity?, J. Clim., 32, 7173-7189, https://doi.org/10.1175/JCLI-D-19-0269.1, 2019.

Dee, D. P., Uppala, S. M., Simmons, A. J., Berrisford, P., Poli, P., Kobayashi, S., Andrae, U., Balmaseda, M. A., Balsamo, G., Bauer, P., Bechtold, P., Beljaars, A. C. M., van de Berg, L., Bidlot, J., Bormann, N., Delsol, C., Dragani, R., Fuentes, M., Geer, A. J., Haimberger, L., Healy, S. B., Hersbach, H., Hólm, E. V., Isaksen, L., Kållberg, P., Köhler, M., Matricardi, M., McNally, A. P., Monge-Sanz, B. M., Morcrette, J.-J., Park, B.-K., Peubey, C., de Rosnay, P., Tavolato, C., Thépaut, J.-N., and Vitart, F.: The ERA-Interim reanalysis: configuration and performance of the data assimilation system, Q. J. R. Meteorolog. Soc., 137, 553-597, https://doi.org/10.1002/qj.828, 2011.

Domeisen, D. I., Butler, A. H., Charlton-Perez, A. J., Ayarzagüena, B., Baldwin, M. P., Dunn-Sigouin, E., Furtado, J. C., Garfinkel, C. I., Hitchcock, P., Karpechko, A. Y., Kim, H., Knight, J., Lang, A. L., Lim, E. P., Marshall, A., Roff, G., Schwartz, C., Simpson, I. R., Son, S. W., and Taguchi, M.: The Role of the Stratosphere in Subseasonal to Seasonal Prediction: 2. Predictability Arising From StratosphereTroposphere Coupling, Journal of Geophysical Research: Atmospheres, 125, https://doi.org/10.1029/2019JD030923, 2020a.

Domeisen, D. I., Butler, A. H., Charlton-Perez, A. J., Ayarzagüena, B., Baldwin, M. P., Dunn-Sigouin, E., Furtado, J. C., Garfinkel, C. I., Hitchcock, P., Karpechko, A. Y., Kim, H., Knight, J., Lang, A. L., Lim, E. P., Marshall, A., Roff, G., Schwartz, C., Simpson, I. R., Son, S. W., and Taguchi, M.: The Role of the Stratosphere in Subseasonal to Seasonal Prediction: 1. Predictability of the Stratosphere, Journal of Geophysical Research: Atmospheres, 125, https://doi.org/10.1029/2019JD030920, 2020b.

Domeisen, D. I. V., Martius, O., and Esteve, B. J.: Rossby Wave Propagation into the Northern Hemisphere Stratosphere: The Role of Zonal Phase Speed, Geophysical Research Letters, 45, 2064-2071, 2018. 
https://doi.org/10.5194/wcd-2021-84

Preprint. Discussion started: 11 January 2022

(C) Author(s) 2022. CC BY 4.0 License.
Weather and

Climate Dynamics

Discussions

Haase, S. and Matthes, K.: The importance of interactive chemistry for stratosphere-troposphere coupling, Atmospheric Chemistry and Physics, 19, 3417-3432, https://doi.org/10.5194/acp-19-3417-2019, 2019.

Harada, Y., Goto, A., Hasegawa, H., Fujikawa, N., Naoe, H., and Hirooka, T.: A Major Stratospheric Sudden Warming Event in January 2009, J. Atmos. Sci., 67, 2052-2069, https://doi.org/10.1175/2009JAS3320.1, 2010.

Hinssen, Y. B. and Ambaum, M. H.: Relation between the 100-hPa heat flux and stratospheric potential vorticity, Journal of the Atmospheric Sciences, 67, 4017-4027, https://doi.org/10.1175/2010JAS3569.1, 2010.

Hitchcock, P. and Shepherd, T. G.: Zonal-Mean Dynamics of Extended Recoveries from Stratospheric Sudden Warmings, J. Atmos. Sci., 70, 688-707, https://doi.org/10.1175/JAS-D-12-0111.1, 2013.

490 Holton, J. R. and Mass, C.: Stratospheric Vacillation Cycles, J. Atmos. Sci., 33, 2218-2225, https://doi.org/10.1175/15200469(1976)033<2218:SVC>2.0.CO;2, 1976.

Jucker, M. and Reichler, T.: Dynamical Precursors for Statistical Prediction of Stratospheric Sudden Warming Events, Geophysical Research Letters, 45, 124-13, https://doi.org/10.1029/2018GL080691, 2018.

Karoly, D. J. and Hoskins, B. J.: Three Dimensional Propagation of Planetary Waves, Journal of the Meteorological Society of Japan. Ser. II, 60, 109-123, https://doi.org/10.2151/jmsj1965.60.1_109, 1982.

Karpechko, A. Y.: Predictability of sudden stratospheric warmings in the ECMWF extended-range forecast system, Monthly Weather Review, 146, 1063-1075, https://doi.org/10.1175/MWR-D-17-0317.1, 2018.

Karpechko, A. Y., Charlton-Perez, A., Balmaseda, M., Tyrrell, N., and Vitart, F.: Predicting Sudden Stratospheric Warming 2018 and Its Climate Impacts With a Multimodel Ensemble, Geophysical Research Letters, 45, 513-538, https://doi.org/10.1029/2018GL081091, 2018.

500 Lee, S. H., Charlton-Perez, A. J., Furtado, J. C., and Woolnough, S. J.: Abrupt Stratospheric Vortex Weakening Associated With North Atlantic Anticyclonic Wave Breaking, Journal of Geophysical Research: Atmospheres, 124, 8563-8575, https://doi.org/10.1029/2019JD030940, 2019.

Limpasuvan, V., Thompson, D. W. J., and Hartmann, D. L.: The Life Cycle of the Northern Hemisphere Sudden Stratospheric Warmings, J. Clim., 17, 2584-2596, https://doi.org/10.1175/1520-0442(2004)017<2584:TLCOTN>2.0.CO;2, 2004.

Limpasuvan, V., Hartmann, D. L., Thompson, D. W. J., Jeev, K., and Yung, Y. L.: Stratosphere-troposphere evolution during polar vortex intensification, J. Geophys. Res. Atmos., 110, https://doi.org/10.1029/2005JD006302, 2005.

Lin, P., Paynter, D., Polvani, L., Correa, G. J. P., Ming, Y., and Ramaswamy, V.: Dependence of model-simulated response to ozone depletion on stratospheric polar vortex climatology, Geophysical Research Letters, 44, 6391-6398, https://doi.org/https://doi.org/10.1002/2017GL073862, 2017.

510 Martius, O., Polvani, L. M., and Davies, H. C.: Blocking precursors to stratospheric sudden warming events, Geophysical Research Letters, 36, https://doi.org/10.1029/2009GL038776, 2009.

Matsuno, T.: Vertical Propagation of Stationary Planetary Waves in the Winter Northern Hemisphere, J. Atmos. Sci., 27, 871-883, https://doi.org/10.1175/1520-0469(1970)027<0871:VPOSPW>2.0.CO;2, 1970.

Matthewman, N. J. and Esler, J. G.: Stratospheric sudden warmings as self-tuning resonances. Part I: Vortex splitting events, Journal of the Atmospheric Sciences, 68, 2481-2504, https://doi.org/10.1175/JAS-D-11-07.1, 2011.

Palmeiro, F. M., Barriopedro, D., García-Herrera, R., and Calvo, N.: Comparing Sudden Stratospheric Warming Definitions in Reanalysis Data, J. Clim., 28, 6823-6840, https://doi.org/10.1175/JCLI-D-15-0004.1, 2015.

Plumb, R. A.: Instability of the distorted polar night vortex: A theory of stratospheric warmings, Journal of the Atmospheric Sciences, 38, 2514-2531, https://doi.org/10.1175/1520-0469(1981)038<2514:IOTDPN>2.0.CO;2, 1981. 
https://doi.org/10.5194/wcd-2021-84

Preprint. Discussion started: 11 January 2022

(C) Author(s) 2022. CC BY 4.0 License.
Weather and

Climate Dynamics

Discussions

Polvani, L. M. and Waugh, D. W.: Upward Wave Activity Flux as a Precursor to Extreme Stratospheric Events and Subsequent Anomalous Surface Weather Regimes, J. Clim., 17, 3548-3554, https://doi.org/10.1175/1520-0442(2004)017<3548:UWAFAA>2.0.CO;2, 2004.

Rao, J., Ren, R., Chen, H., Yu, Y., and Zhou, Y.: The Stratospheric Sudden Warming Event in February 2018 and its Prediction by a Climate System Model, J. Geophys. Res. Atmos., 123, 13,332-13,345, https://doi.org/10.1029/2018JD028908, 2018.

Simpson, I. R., Blackburn, M., and Haigh, J. D.: The role of eddies in driving the tropospheric response to stratospheric heating perturbations, Journal of the Atmospheric Sciences, 66, 1347-1365, https://doi.org/10.1175/2008JAS2758.1, 2009.

Sjoberg, J. P. and Birner, T.: Transient tropospheric forcing of sudden stratospheric warmings, Journal of the Atmospheric Sciences, 69, 3420-3432, https://doi.org/10.1175/JAS-D-11-0195.1, 2012.

Stan, C. and Straus, D. M.: Stratospheric predictability and sudden stratospheric warming events, J. Geophys. Res. Atmos., 114, https://doi.org/10.1029/2008JD011277, 2009.

530 Taguchi, M.: Predictability of major stratospheric sudden warmings of the vortex split type: Case study of the 2002 southern event and the 2009 and 1989 northern events, Journal of the Atmospheric Sciences, 71, 2886-2904, https://doi.org/10.1175/JAS-D-13-078.1, 2014.

Taguchi, M.: Verification of Subseasonal-to-Seasonal Forecasts for Major Stratospheric Sudden Warmings in Northern Winter from 1998/99 to 2012/13, Advances in Atmospheric Sciences, 37, 250-258, https://doi.org/10.1007/s00376-019-9195-6, 2020.

Tripathi, O. P., Charlton-Perez, A., Sigmond, M., and Vitart, F.: Enhanced long-range forecast skill in boreal winter following stratospheric strong vortex conditions, Environ. Res. Lett., 10, 104 007, https://doi.org/10.1088/1748-9326/10/10/104007, 2015.

Tripathi, O. P., Baldwin, M., Charlton-Perez, A., Charron, M., Cheung, J. C. H., Eckermann, S. D., Gerber, E., Jackson, D. R., Kuroda, Y., Lang, A., McLay, J., Mizuta, R., Reynolds, C., Roff, G., Sigmond, M., Son, S. W., and Stockdale, T.: Examining the Predictability of the Stratospheric Sudden Warming of January 2013 Using Multiple NWP Systems, Monthly Weather Review, 144, 1935-1960, https://doi.org/10.1175/MWR-D-15-0010.1, 2016.

540 Vitart, F., Ardilouze, C., Bonet, A., Brookshaw, A., Chen, M., Codorean, C., Déqué, M., Ferranti, L., Fucile, E., Fuentes, M., Hendon, H., Hodgson, J., Kang, H. S., Kumar, A., Lin, H., Liu, G., Liu, X., Malguzzi, P., Mallas, I., Manoussakis, M., Mastrangelo, D., MacLachlan, C., McLean, P., Minami, A., Mladek, R., Nakazawa, T., Najm, S., Nie, Y., Rixen, M., Robertson, A. W., Ruti, P., Sun, C., Takaya, Y., Tolstykh, M., Venuti, F., Waliser, D., Woolnough, S., Wu, T., Won, D. J., Xiao, H., Zaripov, R., and Zhang, L.: The subseasonal to seasonal (S2S) prediction project database, Bulletin of the American Meteorological Society, 98, 163-173, https://doi.org/10.1175/BAMS-D-16-0017.1, 2017. 